\title{
Carrier mode selective working point and side band imbalance in LIGO I
}

\author{
Erika D'Ambrosio and William Kells \\ California Institute of Technology, Pasadena, California 91125, USA
}

(Received 27 February 2006; published 5 June 2006)

\begin{abstract}
In gravitational wave interferometers, the input laser beam is phase modulated to generate radiofrequency side bands that are used to lock the cavities. The mechanism is the following: the frequency of the side bands and the carrier is chosen in such a way that their response to small changes of the longitudinal degrees of freedom is different. This difference is therefore monitored and it serves as an error signal for controlling the optical cavity lengths, as they are linearly related to the set of observed phases between carrier and side bands. Among the others, one longitudinal degree of freedom is optimally sensitive to the space-time distortions propagating through the cosmos, as predicted by the general theory of relativity. The observation of the astrophysical signal relies on the measurement of that specific degree of freedom. The entire problem is more complex when the transverse degrees of freedom are taken into account, because the relative phase between the fields also depends on their overlap. In order to establish an unambiguous relation between length changes and phase measurements, there must be one circulating optical mode and the only difference between carrier and side bands must be their amplitude. We will show that the variability of the transverse degrees of freedom and their different actions on carrier and side band fields puts a severe limit on this assumption. Unless the system is made of perfect and perfectly matched optical cavities, it is never governed by one unique coherent state and any adjustment of the optical lengths results from a compromise between the lengths that are optimal for the carrier field and the side band ones. Such a compromise alters the correspondence between error signals and cavity lengths, calculated in the one-dimensional treatment. We assess the strength of this effect and relate it to the sensitivity of the instrument (which relies on the reconstruction of that correspondence) in realistic circumstances.
\end{abstract}

DOI: 10.1103/PhysRevD.73.122002

PACS numbers: 04.80.Nn, 07.60.Ly, 41.85.-p, 42.60.-v

\section{INTRODUCTION}

Phase modulation of a single mode laser source was introduced as a convenient method of null servo locking optical cavities to a source. High sensitivity to the cavity carrier resonance is achieved by optical heterodyne detection with side bands generated by phase modulation: the radio-frequency current measured by a photodiode in cavity reflection is null exactly at the carrier resonance.

Modulation phase and amplitude fluctuations do not affect this null point, but any imbalance between the two side bands detected by the photodiode adds some new term to the radio-frequency current, that is additional noise in the servo signal. The effect is proportional to the imbalance and also causes a shift of the carrier resonance null [1].

In this paper, we identify and study a variety of physical causes of side band imbalance. We clarify first the traditional notion that a sufficiently perfect optical configuration is intrinsically balanced for enough low frequency. With such an ideal configuration as the unperturbed starting point of our analysis, we introduce a variety of typical imperfections, examining their impact on the side band balance. We find that the balance is maintained in several realistic configurations and therefore the operating point is robust, against very small residual optical alignment and aberration imperfections. Since our focus is on gravitational wave interferometers, we assume some strong constraints that are typical of the regime currently encountered in LIGO and similar detectors. One of these is that only the first modulation side bands are relevant; that means the ones at frequency $\nu^{ \pm}=\nu^{0} \pm \nu^{\text {mod }}$. The frequency $\nu^{\text {mod }}$ is assumed to be small enough, that the optical properties of all elements are identical over the frequency range $\left[\nu^{0}-\right.$ $\left.\nu^{\text {mod }}, \nu^{0}+\nu^{\text {mod }}\right]$. In our treatment we consider diffraction losses to be negligible. This also implies that we can assume all beams and cavity modes strictly Gaussian, their transverse edge not being determined by finite aperture effects. We engage optical elements of highest quality and precision [2].

One related and crucial assumption is that the phase modulation of the source laser beam generates side band fields that are identical to the carrier field.

This geometrical feature is quite essential since, while the actual modulation frequency is a free parameter and can be changed to adjust the relative phase between the side bands and the carrier, there is no spatial adjustment that we can tune to compensate for the difference in the transverse intensity profile. This is determined by the interferometer configuration itself. We will examine the relation between the geometry of a complex resonator and its normal modes, establish a rigorous correspondence between optimal working point and minimum spurious contributions to the radio-frequency photodetector current we use as physical observable quantity, and analyze the typical circumstances underneath recoverable and unrecoverable contaminations of the gravitational wave signal. 


\section{ONE-DIMENSIONAL CONFIGURATION}

The familiar two spherical mirror cavity will serve to introduce our approach and interpretation of side band balance. We choose for illustrative purposes an optical resonator with a flat input mirror as in Fig. 1. We assume a family of Laguerre-Gaussian or Hermite-Gaussian functions, that we identify by the beam waist and location [3].

The light frequency is also a relevant parameter, because the side bands would correspond to different eigenmode families, the waist size being a function of the frequency of interest

$$
w_{0}(\nu) \propto \nu^{-1 / 2} .
$$

We will ignore such a difference and the imbalance it implies, as the range we are interested in is typically $\nu^{\text {mod }} / \nu^{0} \leq 10^{-7}$. This condition on the two sets of modes $\Psi_{i}^{ \pm}$is not sufficient to guarantee balance.

The side band normal modes are excited by the input beam, as it is illustrated in Fig. 1. We will assume that the input beam itself is balanced and the first harmonic components are equal. In this case the coupling between the eigenmodes $\Psi_{i}^{ \pm}$and the input field $\psi_{\mathrm{IN}}^{+}$is the same.

Given the above constraint on the driving beam, the response of the side bands is now entirely governed by the eigenvalues $\lambda_{i}^{ \pm}$. Since we have assumed all the optical elements are frequency independent, the absolute value of the eigenvalues is the same $\left|\lambda_{i}^{+}\right|=\left|\lambda_{i}^{-}\right|$. When the cavity is lossless $\left|\lambda_{i}^{ \pm}\right|=1$. More generally when the losses are scalar quantities, such as in the case of uniform transmittance of the optics

$$
\left|\lambda_{i}^{ \pm}\right|=1-\mathcal{L} \quad \forall i
$$

with $\mathcal{L}$ the total loss. Therefore the side band optical modes can only be distinguished by the phase $\arg \left[\lambda_{i}^{ \pm}\right]$. The fast component of this phase is a plane wave term $\propto$

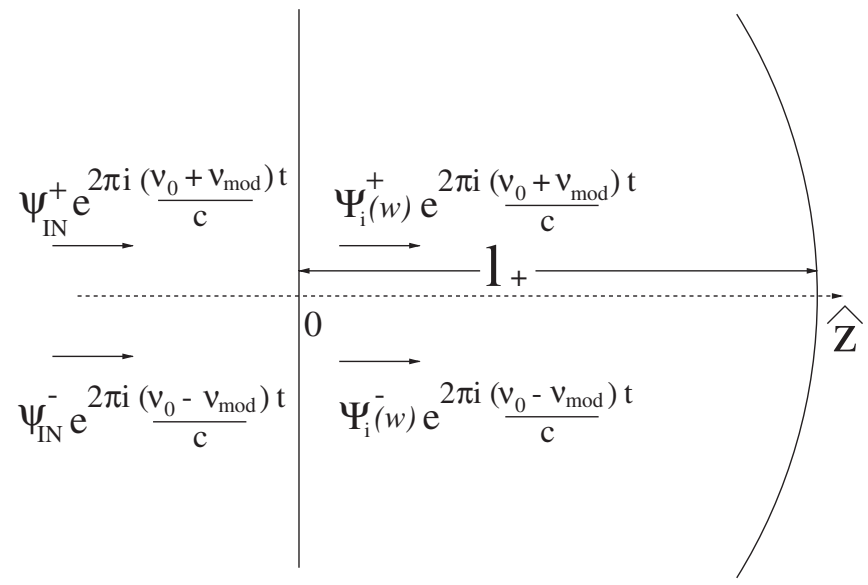

FIG. 1. The first side bands produced by phase modulation of the laser source are typically identical for the radio-frequency range we are interested in, so that $\psi_{\mathrm{IN}}^{+}=\psi_{\mathrm{IN}}^{-}$. We also assume $\Psi_{i}^{+}=\Psi_{i}^{-}$for the normal modes. $\nu^{ \pm} z$. The eigenmode equivalence

$$
\lambda_{i}^{+}=\lambda_{i}^{-} \Longleftrightarrow \arg \left[\lambda_{i}^{+}\right]=\arg \left[\lambda_{i}^{-}\right] \quad(\bmod 2 \pi)
$$

is generally equivalent to

$$
\frac{2 \pi \nu^{\bmod }}{c} 2 l_{+}=\pi, 2 \pi, 3 \pi, \ldots
$$

with $c$ the speed of light and $l_{+}$the cavity length. This also implies the resonant or antiresonant condition for any modal component of the side bands, when the same modal component of the carrier $\Psi_{i}^{0}$ is resonant. Exact tuning of $\nu^{\text {mod }}$ is required to fulfil this requirement. The ideal normal situation would be the antiresonant case, since it allows servo locking error detection. Simultaneous resonance is practically useless since it implies side bands and carrier are not distinguishable. An interesting property is that the SB balance can be restored by means of $\nu^{0}$. Applying the proper adjustment

$$
\arg \left[\lambda_{i}^{0}\right]=\frac{2 \pi \nu^{0}}{c} 2 l_{+}-2 \theta_{i}^{G}=0 \quad(\bmod \pi)
$$

where $\theta_{i}^{G}$ is the slow component of the phase. The above condition entails $\lambda_{i}^{+}=\lambda_{i}^{-*}$. This more general criterion for side band balance only holds for the eigenmodes resonating or antiresonating at $\nu^{0}$ because of the contribution due to the Gouy phase $\theta_{i}^{G}$.

If the side band eigenvalues are complex conjugate quantities, the gain is the same and therefore the field magnitude is identical. This is the nominal ideal for a variety of practical purposes. It implies conditions on the carrier that coincide with the working point of most optical systems. When systems are more complex than a nondegenerate two mirror cavity, the carrier being resonant or antiresonant does not suffice for making the side bands effectively balanced.

\section{Optimally coupled cavities}

By inserting an ideal and partially reflecting septum between the end mirrors of a simple cavity, we obtain the double cavity shown in Fig. 2. For an arbitrary septum the eigenmode set is not stable under frequency shifts. In general there are two waves traveling in opposite direction at any time. When the septum exactly coincides with the wave front of the original normal modes, at any location inside this more complex optical configuration the transverse light distribution is the same for the traveling wave solutions and does not depend on $l_{+}$and $L_{+}$. This is crucial for all coupled resonators and when this matching condition is not satisfied, we find different sets of eigenmodes for each pair $\left\{l_{+}, L_{+}\right\}$that implies no mode resonance can be established.

The ideal situation for gravitational wave interferometers is exactly matched coupled cavities, so that the circulating modes are the same as the eigenmodes of each single cavity. 


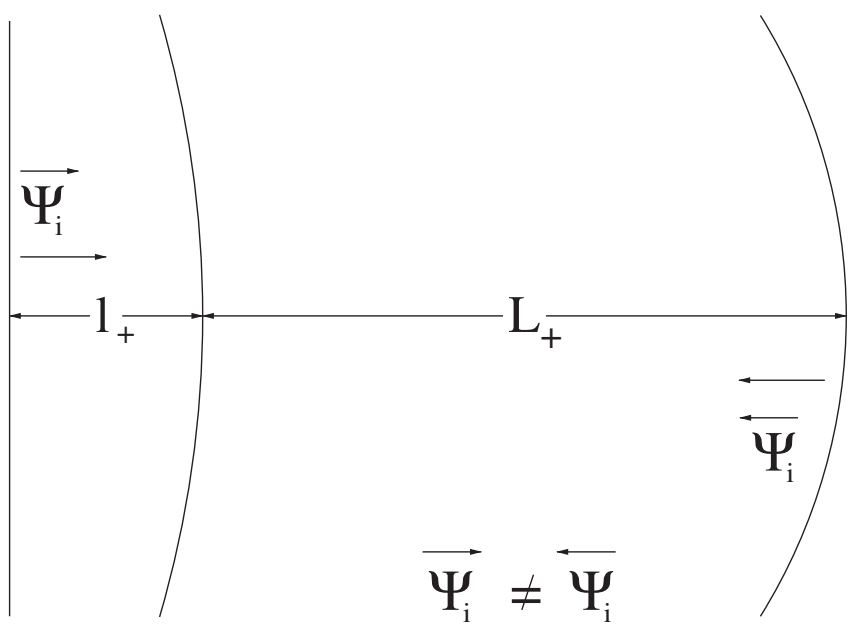

FIG. 2. Solving the wave equation with the border conditions corresponding to a double cavity results in eigenfunctions, with two opposite traveling components and a relative weight depending on the coupling between the cavities, which in general is a function of $l_{+}$and $L_{+}$unless these are separable variables.

Under this restriction, our previous statements on the description of the side band fields apply again.

In particular, the criteria we have established for side band balance must be satisfied by each cavity.

For example, the condition on the carrier being resonant or antiresonant must be extended to each cavity and it makes $\lambda_{i}^{+}=\lambda_{i}^{-*}$.

Similarly, the simultaneous conditions

$$
\begin{aligned}
& \frac{2 \pi \nu^{\mathrm{mod}}}{c} 2 l_{+}=\pi, 2 \pi, 3 \pi, \ldots \\
& \frac{2 \pi \nu^{\mathrm{mod}}}{c} 2 L_{+}=\pi, 2 \pi, 3 \pi, \ldots
\end{aligned}
$$

correspond to $\lambda_{i}^{+}=\lambda_{i}^{-}$. Since there are two phases to be adjusted, with no restriction on the lengths there is now no special tuning of $\nu^{0}$ that can make the carrier resonate or antiresonate in each cavity. In this case the two lengths must be separately tuned. Alternatively they must be an integer multiple of $c /\left(4 \nu^{\mathrm{mod}}\right)$. Generally side band balance can be achieved by any combination of a macroscopic and a microscopic condition; that is the carrier frequency can be adjusted according to one of the cavities and $\nu^{\text {mod }}$ can be tuned to satisfy the length condition for the other cavity.

As an illustrative example, we may regard LIGO as being equivalent to a double cavity. The lengths $\left\{l_{+}, L_{+}\right\}$ are tuned for the carrier to resonate in all cavities. The macroscopic condition on $\left\{l_{+}, L_{+}\right\}$is also very close to its perfect realization; it is desirable to avoid simultaneous resonance of second order side band fields. The global length sensing and control of a system of multiple coupled optical resonators is a vast problem and further references can be found in [4].

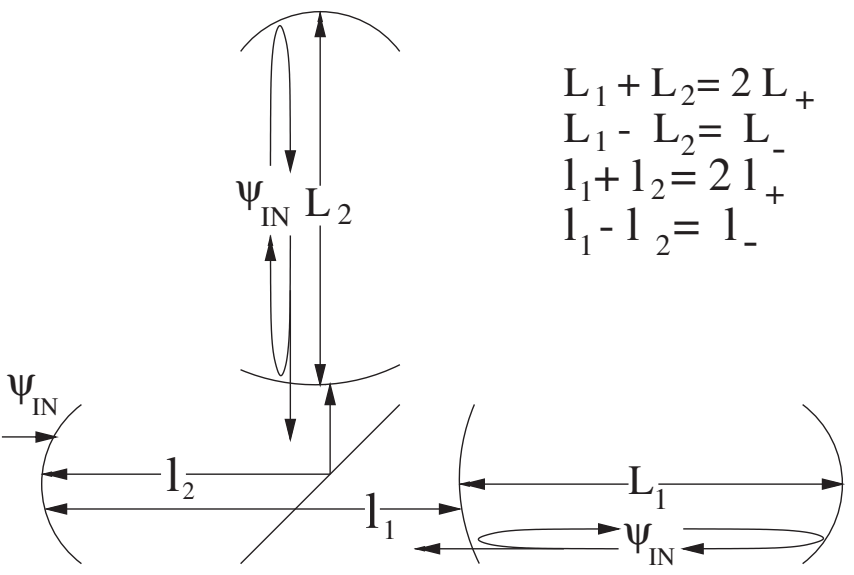

FIG. 3. The lengths $\left\{L_{1}, L_{2}, l_{+}\right\}$are multiples of $c /\left(4 \nu^{\bmod }\right)$. When the carrier resonates in the arm cavities, the side bands become antiresonant and they are therefore only sensitive to the features of the recycling cavity.

The longitudinal degrees of freedom in all gravitational wave interferometers are four as illustrated in Fig. 3. The differential length $L_{1}-L_{2}$ is measured by heterodyne detection at the dark port of the beam splitter. The escaping carrier light proportional to $L_{1}-L_{2}$ beats against the side bands. As the carrier resonates in the arm cavities and the side bands antiresonate, their sensitivity to $L_{-}=L_{1}-L_{2}$ is minimum. Hence the side bands are a good fixed reference for the variations induced by $L_{-}$on the carrier. The amount of side band light available at the dark port is due to $l_{1}-l_{2}=l_{-} \neq 0$. If the beam splitter is kept fixed on the carrier dark fringe, the side bands are exactly in balance since they experience the same loss at the dark port. There are three macroscopic length conditions satisfied in LIGO and they correspond to $\left\{L_{1}, L_{2}, l_{+}\right\}$that are integer multiples of $c /\left(4 \nu^{\mathrm{mod}}\right)$. There are four microscopic length conditions; three of them regard the carrier resonance and one corresponds to

$$
\frac{2 \pi \nu^{0}}{c} 2 l_{-}=0 \quad(\bmod 2 \pi)
$$

so that when all degrees of freedom are properly locked, the entire system can be considered optimally operating.

\section{MODAL ABERRATIONS}

A standard configuration has now been established, with respect to which there is a complete symmetry in the responsive behavior of the side bands. When the cavities are all perfectly matched, there is only one spatial mode circulating everywhere. To best couple light into the interferometer, the input beam needs to have that same spatial distribution. For the macroscopic conditions we have illustrated above, the side bands are always balanced unless the beam splitter is not locked on the dark fringe of the carrier. More interesting is exploring the effect of transverse dis- 
tortions. Without loss of generality we assume the perturbed elements are the back of the Michelson optics, with the carrier beam defined by the surface figures of the Fabry-Perot optics. Since the laser beam is kept fixed and matched to the carrier mode, any distortion in the Michelson cavity has no effect on the carrier to first order in the perturbation (if the interferometer is in full lock) while the side band fields are rather affected and the perturbation they undergo is the focus of our analysis. The eigenmodes and the eigenvalues of the Michelson cavity determine the side band fields. We eliminate the arm resonators since they represent balanced reflectivities and keep fixed the input beam mode. The unperturbed system is therefore defined by perfect optical match with the input and distortions are defined with respect to its phase front [5-7]. In this matrix and state vector formalism, the perturbed cavity eigenmodes can be expressed as the sum of the input mode plus a perturbative series of terms depending on some measure of the distortions $\sim \alpha, \sim \alpha^{2}, \ldots$

The perturbative action of each distorted mirror is expressed as a scattering matrix $\mathbf{m}(\alpha)$. Each element in $\mathbf{m}(\alpha)$ corresponds to the intermodal coupling due to the deviations from exact matching, with respect to the unperturbed original basis.

We study the conditions for side band balance in the perturbative case of two different Michelson optics. Were these perfectly identical and were the system stable, this would be the same as a single resonator and its set of eigenmodes would be unambiguously identified.

In addition, we assume

$$
l_{1} \simeq l_{2} \simeq l_{+} \quad l_{+} \equiv \frac{l_{1}+l_{2}}{2}
$$

using the notations of Fig. 3. This means that the modes of a branch are identical to the modes of the other. The differential length $l_{-}=l_{1}-l_{2}$ must therefore be much smaller than the corresponding Rayleigh length, that is the typical length, over which the modes would change significantly and the overlap mismatch becomes relevant.

With respect to this ideal interferometer, we start analyzing the consequences of different Michelson optics. Since any common perturbation would equally affect the two branches and their optical modes, we choose the common interferometer as our starting unperturbed configuration, whatever it is. In this basis, the two Michelson optics are described by the same and opposite perturbative parameter $\alpha$. There is a different coupling and $\alpha$ is proportional to the magnitude of the excitation of the higher order modes, for each branch. We assume that energy is conserved and introduce a unitary matrix $\mathbf{m}(\alpha)$ for one perturbation and $\mathbf{m}(-\alpha)$ for its inverse.

The length $l_{-}=l_{1}-l_{2}$ is now no longer a separable quantity. Different modes are excited in the two branches and the overall interference depends on their complex combination of modes.
We use a simple example to illustrate the fundamental difference between this mechanism and the unperturbed situation. We assume that the Rayleigh length is much larger than the common length $l_{+}$. The bright port of the beam splitter consists of the combination

$$
\psi_{\mathrm{BP}}(\delta)=\frac{e^{-i \delta} \mathbf{m}(\alpha)+e^{i \delta} \mathbf{m}(-\alpha)}{2} \psi_{\mathrm{IN}} \quad \delta \propto l_{-}
$$

and we see that the dependence on $\delta$ is not a constant we can factorize.

On the contrary $\delta$ can even determine the shape of the stationary field. In this extreme but simple case we use for illustrative purpose, we suppose we find the eigensolutions of

$$
\mathbf{m}(\alpha) \psi_{i}(\alpha)=\mu_{i}(\alpha) \psi_{i}(\alpha) \quad \mathbf{m}(-\alpha)=\mathbf{m}^{-1}(\alpha)
$$

and use them to find the optimal input beam $\psi_{\mathrm{IN}}=\psi_{\mathrm{BP}}(\delta)$. For any $\delta \propto l_{-}$the best choice corresponds to the following eigenmode

$$
\psi_{\mathrm{IN}}=\psi_{i}(\alpha) ; \quad \cos \left(\delta+\arg \left[\mu_{i}\right]\right)=\max
$$

which means its form is determined by $\delta \propto l_{-}$. Note that $l_{+}$can still be separately adjusted.

As the role of the differential length becomes unavoidably entangled with the transverse degrees of freedom, the sufficient conditions for side band balance must be thoroughly revisited. In addition, since the indistinguishability of such conditions and the carrier dark port condition is used to maintain the beam splitter on the carrier dark fringe in all LIGO detectors, we will contextually address the question of the "optimal" beam splitter position. This is not a trivial problem, because the role of the differential length and the concept itself of dark fringe must be redefined in terms of state vectors.

\section{A. Nearly degenerate optical cavities}

We examine again the nearly degenerate limit of the power recycling cavity as an illustrative simple case, because the extension of the bright and dark port concept to the space of state vectors does not depend on the specification of the bright and dark port physical location when $\mathbf{p}=\mathbb{1}$. Later we will see that, unless the system eigenmodes are the familiar Laguerre-Gaussian or HermiteGaussian functions, the location of those two and other observables is relevant and we can no longer assume ubiquitous phase front equivalence of the system normal modes.

We have two problems to solve: we want efficient coupling of the input beam to the optical resonator and no light loss at the antisymmetric port of the beam splitter. Solving (1) for $\psi_{\mathrm{BP}} \propto \psi_{\mathrm{IN}}$ corresponds to the former condition and imposing $\left|\psi_{\mathrm{BP}}\right|^{2}=\left|\psi_{\mathrm{IN}}\right|^{2}$ to the latter. The two can be simultaneously satisfied; the input beam must coincide with one of the solutions of (2) which are independent from $\delta$. For any $\delta$ the carrier and side band transverse 
distributions are the same. When the antisymmetric port signal is zero, the two side bands are characterized by the same loss and their intracavity fields are identical. This is described by

$$
\left|\cos \left(\delta+\arg \left[\mu_{i}\right]\right)\right|^{2}=1 \quad \text { with } \delta=\delta^{0},
$$

which imposes the conservation of energy associated to the bright port action

$$
\mathcal{B} \mathcal{P}(\delta) \psi_{i}(\alpha)=\cos \left(\delta+\arg \left[\mu_{i}\right]\right) \psi_{i}(\alpha)
$$

at the carrier frequency.

For the side bands $\delta=\delta^{0} \pm \delta^{\mathrm{mod}}$. We see that if $\delta^{0}$ satisfies the carrier bright port condition, the dark port is identically zero and the side bands are equal. This exact and apparently obvious correspondence does not hold for any multidimensional case, which poses the issue of what the proper generalization should be. In general, the two requirements on the optimal input coupling and dark fringe condition we have above discussed may not admit one exact consistent solution.

Furthermore, identifying the approximation corresponding to such a solution is not straightforward and two complicacies occur: the optical modes depend on the differential phase and in this basis the dark port is not a diagonal operator.

The former generalization implies the stationary side band fields are not the same as their input. As their modal content can be different, equal side band loss does not suffice to make the intracavity fields identical and the exact correspondence between dark port condition and side band balance fails; the eigenvalue of interest is not the only quantity we must account for and any comparison becomes vectorial in character. The generalization of that correspondence is also related to the other generalization between the carrier bright and dark port conditions, that becomes a tensorial problem.

Before we enter the study of an approximate multidimensional solution of such a composite set of conditions, we want to outline the overall assumption of an ideal beam splitter, which otherwise would make the above correspondences even more difficult to treat.

In addition, as we are closely focused on the detection of gravitational waves and the impact of side band imbalance on the ultimate performance of LIGO, our next analysis must always be intended aimed to improve the operating point of LIGO and alike systems [8].

\section{B. Stable common geometrical configuration}

Referring to Fig. 4 the case $\mathbf{p} \neq \mathbb{1}$ is now studied.

We start from the generalization of (1). The common configuration is stable, which thus identifies

$$
\mathbf{p}_{n m}=\delta_{n m} \exp \left[-i \theta_{n}^{G}\right]
$$

where $\theta_{n}^{G}$ are the unperturbed Gouy phases. The action of $l_{-}$is entangled with the differential optical aberrations, in a

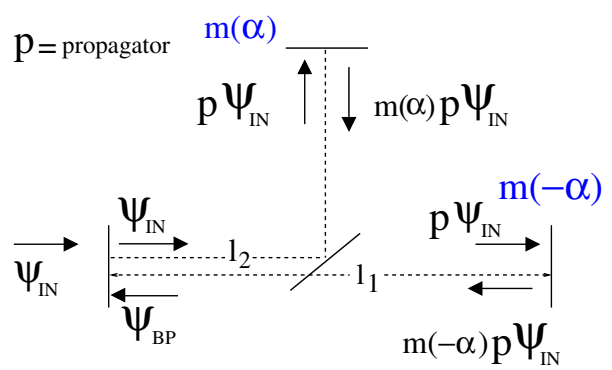

FIG. 4 (color online). The common length and the differential length should satisfy resonant and dark port conditions. Unless the two Michelson optics are identical, these two conditions are not at all independent from each other because the transverse light intensity of the symmetric combination of the reflected beams does not simply scale with their relative phase $\propto$ $\cos 2 \pi \nu 2 l_{-} / c$, but the shape itself is affected. In other words, $l_{-}$determines the eigenmodes and the eigenvalues of the system.

way that affects the solutions of the equation

$$
\psi_{\mathrm{BP}}(\delta)=\frac{\mathbf{p} e^{-i \delta} \mathbf{m}(\alpha) \mathbf{p}+\mathbf{p} e^{i \delta} \mathbf{m}(-\alpha) \mathbf{p}}{2} \psi_{\mathrm{IN}}
$$

when we impose $\psi_{\mathrm{IN}} \propto \psi_{\mathrm{BP}}$. In (4) an overall common phase is always intended factored out and neglected. We study the solutions of

$$
\lambda_{i}(\delta) \psi_{i}(\delta)=\frac{\mathbf{p} e^{-i \delta} \mathbf{m}(\alpha) \mathbf{p}+\mathbf{p} e^{i \delta} \mathbf{m}(-\alpha) \mathbf{p}}{2} \psi_{i}(\delta)
$$

to design the form of the input $\psi_{\mathrm{IN}}$ that optically matches the system. In this general case, changing the value of the differential length results in different normal modes. We emphasize this dependence in the eigenvector matrix

$$
\mathbf{M}_{i j}(\delta)=\left[\psi_{j}(\delta)\right]_{i}
$$

where the components $[\ldots]$ are computed in the unperturbed basis. We also want to minimize the optical loss and this is done by adjusting $\delta$. This composite problem is therefore equivalent to designing the input field as one of the eigenmodes, that should also correspond to the most constructive bright port combination $\delta$ can allow. The resulting bright port beam coherently overlaps with the incoming $\psi_{\mathrm{IN}} \propto \psi_{\mathrm{BP}}$ and power builds up. We are using all the power we can coherently overlap, when the dark port contains no fundamental mode loss. We use $\mathcal{B P}(\delta)$ to represent the overall operator in (4). The corresponding antisymmetric combination is denoted by $\mathcal{D P}(\delta)$.

Motivated by the design of the input beam, the bright port has been identified at the input mirror location. We also assume that the dark and bright port are at the same optical distance from the beam splitter, that is the antisymmetric and symmetric combinations are propagated by the same amount. We designate the former by the operator

$$
\mathcal{D} \mathcal{P}(\delta)=\frac{\mathbf{p} e^{-i \delta} \mathbf{m}(\alpha) \mathbf{p}-\mathbf{p} e^{i \delta} \mathbf{m}(-\alpha) \mathbf{p}}{2}=i \frac{\mathrm{d}}{\mathrm{d} \delta} \mathcal{B} \mathcal{P}(\delta) .
$$


From the mathematical point of view, this definition of the dark port operator simplifies the treatment of the simultaneous solution of bright and dark port condition, meaning the microscopic differential length coincides with the dark fringe of the carrier stationary field. This one is assigned as our input beam

$$
\psi_{1}\left(\delta^{0}\right) ;\left.\quad \frac{\mathrm{d}}{\mathrm{d} \delta}\left[\mathbf{M}^{-1}(\delta) \mathcal{B P}(\delta) \mathbf{M}(\delta)\right]_{11}\right|_{\delta=\delta^{0}}=0 .
$$

Here 1 conventionally designates the fundamental eigenmode $\psi_{1}(\delta)$. We assume a continuous dependence on $\delta$ of eigenvectors and eigenvalues. This allows us to slightly modify the differential length and derive the form

$$
\mathbf{M}^{-1}(\delta) \mathcal{B} \mathcal{P}(\delta) \mathbf{M}(\delta)=\left(\begin{array}{ccc}
\lambda_{1}(\delta) & 0 & \cdots \\
0 & \lambda_{2}(\delta) & \cdots \\
\vdots & \vdots & \ddots
\end{array}\right)
$$

where $\lambda_{1}(\delta)$ is the quantity of interest. The above condition can be expanded and simplified

$$
\begin{aligned}
\frac{\mathrm{d}}{\mathrm{d} \delta}[ & \left.\mathbf{M}^{-1}(\delta) \mathcal{B} \mathcal{P}(\delta) \mathbf{M}(\delta)\right]_{11} \\
= & {\left[\frac{\mathrm{d} \mathbf{M}^{-1}(\delta)}{\mathrm{d} \delta} \mathcal{B} \mathcal{P}(\delta) \mathbf{M}(\delta)+\mathbf{M}^{-1}(\delta) \frac{\mathrm{d} \mathcal{B} \mathcal{P}(\delta)}{\mathrm{d} \delta} \mathbf{M}(\delta)\right.} \\
& \left.+\mathbf{M}^{-1}(\delta) \mathcal{B} \mathcal{P}(\delta) \frac{\mathrm{d} \mathbf{M}(\delta)}{\mathrm{d} \delta}\right]_{11} \\
= & -i\left[\mathbf{M}^{-1}(\delta) \mathcal{D} \mathcal{P}(\delta) \mathbf{M}(\delta)\right]_{11}=0
\end{aligned}
$$

thanks to the identity

$$
\mathbf{M}^{-1}(\delta) \mathbf{M}(\delta)=1 \longrightarrow \frac{\mathrm{d}}{\mathrm{d} \delta}\left[\mathbf{M}^{-1}(\delta) \mathbf{M}(\delta)\right]_{11}=0
$$

and the familiar properties of $\delta_{m n}$ the Kronecker delta symbol. Since the dark port contains no input beam light, the contrast of the interferometer is the best achievable. This corresponds to the intuitive condition that the sym- metric combination is constructive for the fundamental mode and destructive for the excited ones, in exact opposite to the antisymmetric combination. When the perturbation excites one single mode, the system performs a mode transformation and were the output and input switched, the reverse transformation would operate and the input and output field would now be the excited mode and the fundamental one. In analogy to the simple optical resonator, driving a system by an excited mode is not an optimal choice, although feasible.

We now go back to the problem of the impact of the dark port condition on the side band balance. After one roundtrip $\delta=\delta^{0} \pm \delta^{\text {mod }}$ mixes the input beam with the excited modes, as their destructive interference is no longer precise, so that

$$
\begin{aligned}
\psi_{\mathrm{BP}}^{ \pm} & =\frac{\mathbf{p} e^{-i\left(\delta^{0} \pm \delta^{\mathrm{mod}}\right)} \mathbf{m}(\alpha) \mathbf{p}+\mathbf{p} e^{i\left(\delta^{0} \pm \delta^{\mathrm{mod}}\right)} \mathbf{m}(-\alpha) \mathbf{p}}{2} \psi_{\mathrm{IN}} \\
& =\left(\cos \delta^{\mathrm{mod}} \mathcal{B} \mathcal{P}\left(\delta^{0}\right) \mp i \sin \delta^{\mathrm{mod}} \mathcal{D} \mathcal{P}\left(\delta^{0}\right)\right) \psi_{\mathrm{IN}}
\end{aligned}
$$

is recirculated and is subject to the next differential scattering, so that an incremental effect is produced

$$
\begin{aligned}
& \mp i \sin \delta^{\bmod } \mathcal{D} \mathcal{P}\left(\delta^{0}\right)-\sin \delta^{\bmod } \mathcal{D} \mathcal{P}\left(\delta^{0}\right) \\
& \times \frac{1}{1-\cos \delta^{\bmod } \mathcal{B P}\left(\delta^{0}\right)} \sin \delta^{\bmod } \mathcal{D} \mathcal{P}\left(\delta^{0}\right) \\
& \quad \pm i \sin \delta^{\bmod } \mathcal{D} \mathcal{P}\left(\delta^{0}\right) \frac{1}{1-\cos \delta^{\bmod } \mathcal{B P}\left(\delta^{0}\right)} \\
& \quad \times \sin \delta^{\bmod } \mathcal{D} \mathcal{P}\left(\delta^{0}\right) \frac{1}{1-\cos \delta^{\bmod } \mathcal{B P}\left(\delta^{0}\right)} \\
& \quad \times \sin \delta^{\bmod } \mathcal{D} \mathcal{P}\left(\delta^{0}\right)+\ldots
\end{aligned}
$$

as described in Appendix A.

Applying standard summation techniques, we first consider the following series

$$
\begin{aligned}
\mathcal{S}^{ \pm}= & {\left[\mathbf{M}^{-1}\left(\delta^{0}\right) \sin \delta^{\bmod } \mathcal{D} \mathcal{P}\left(\delta^{0}\right) \mathbf{M}\left(\delta^{0}\right)\right]_{1 k} \frac{1}{1-\cos \delta^{\bmod } \lambda_{k}\left(\delta^{0}\right)}\left[\mathbf{M}^{-1}\left(\delta^{0}\right) \sin \delta^{\bmod } \mathcal{D} \mathcal{P}\left(\delta^{0}\right) \mathbf{M}\left(\delta^{0}\right)\right]_{k 1} } \\
& \mp i\left[\mathbf{M}^{-1}\left(\delta^{0}\right) \sin \delta^{\bmod } \mathcal{D} \mathcal{P}\left(\delta^{0}\right) \mathbf{M}\left(\delta^{0}\right)\right]_{1 k} \frac{1}{1-\cos \delta^{\bmod } \lambda_{k}\left(\delta^{0}\right)} \\
& \times\left[\mathbf{M}^{-1}\left(\delta^{0}\right) \sin \delta^{\bmod } \mathcal{D} \mathcal{P}\left(\delta^{0}\right) \mathbf{M}\right]\left(\delta^{0}\right)_{k m} \frac{1}{1-\cos \delta^{\bmod } \lambda_{m}\left(\delta^{0}\right)}\left[\mathbf{M}^{-1}\left(\delta^{0}\right) \sin \delta^{\bmod } \mathcal{D} \mathcal{P}\left(\delta^{0}\right) \mathbf{M}\left(\delta^{0}\right)\right]_{m 1}+\ldots
\end{aligned}
$$

where repeated indices designate internal sums. They are $k, m, \ldots \neq 1$ and $\perp$ refers to the subspace of state vectors they span. Using this notation

$$
\begin{aligned}
\mathcal{S}^{ \pm}= & {\left[\mathbf{M}^{-1}\left(\delta^{0}\right) \sin \delta^{\bmod } \mathcal{D} \mathcal{P}\left(\delta^{0}\right) \mathbf{M}\left(\delta^{0}\right)\right.} \\
& \left.\times\left(\frac{1}{1-\left.\mathbf{M}^{-1}\left(\delta^{0}\right)\left(\cos \delta^{\bmod } \mathcal{B} \mathcal{P}\left(\delta^{0}\right) \mp i \sin \delta^{\bmod } \mathcal{D} \mathcal{P}\left(\delta^{0}\right)\right) \mathbf{M}\left(\delta^{0}\right)\right|_{\perp}}\right) \mathbf{M}^{-1}\left(\delta^{0}\right) \sin \delta^{\bmod } \mathcal{D} \mathcal{P}\left(\delta^{0}\right) \mathbf{M}\left(\delta^{0}\right)\right]_{11}
\end{aligned}
$$

is the perturbative variation of the fundamental mode. Since we are interested in the overlap of the stationary side band and carrier fields, we evaluate the inclusive final sum, as 


$$
\begin{aligned}
& {\left[\mathbf{M}^{-1}\left(\delta^{0}\right) \frac{1}{1-\cos \delta^{\bmod } \mathcal{B} \mathcal{P}\left(\delta^{0}\right) \pm i \sin \delta^{\bmod } \mathcal{D} \mathcal{P}\left(\delta^{0}\right)} \mathbf{M}\left(\delta^{0}\right)\right]_{11}} \\
& =\frac{1}{1-\cos \delta^{\bmod } \lambda_{1}\left(\delta^{0}\right)}-\frac{1}{1-\cos \delta^{\bmod } \lambda_{1}\left(\delta^{0}\right)} \mathcal{S}^{ \pm} \frac{1}{1-\cos \delta^{\bmod } \lambda_{1}\left(\delta^{0}\right)} \\
& \quad+\frac{1}{1-\cos \delta^{\mathrm{mod}} \lambda_{1}\left(\delta^{0}\right)} \mathcal{S}^{ \pm} \frac{1}{1-\cos \delta^{\mathrm{mod}} \lambda_{1}\left(\delta^{0}\right)} \mathcal{S}^{ \pm} \frac{1}{1-\cos \delta^{\mathrm{mod}} \lambda_{1}\left(\delta^{0}\right)}+\ldots
\end{aligned}
$$

that is the second and last step of the summation. The above can be written as

$$
\frac{1}{1-\cos \delta^{\bmod } \lambda_{1}\left(\delta^{0}\right)+\mathcal{S}^{ \pm}}
$$

where $\mathcal{S}^{ \pm}$is the term responsible for side band asymmetries. This already contains all the information we need to discuss the imbalance problem.

We see right away that, as a result of our generalized definition of operating point, the portion of the side bands that coherently overlaps with the driving beam and therefore builds up power, is about the same and the magnitude of the first term generating discrepancy is $\mathcal{O}\left(\alpha^{3}\right)$ or smaller. The real part of $\mathcal{S}^{ \pm}$affects the storage time. The side band losses are no longer uniquely due to the macroscopic length asymmetry; moreover the combined effect of length asymmetry and differential aberrations can also contribute a phase shift from resonance. We will address these points later, when the strength of the perturbation will be compared with the spacing the unperturbed eigenvalues are separated by, our conclusions being based on the familiar assumptions of perturbative expansion.

Before we enter this more quantitative study in our analysis and examine some examples, we want to demonstrate a few properties our definition of bright and dark port makes robustly propagated from the unidimensional representation to the multidimensional one. The perturbed resonant mode is a complex combination of HermiteGaussian or Laguerre-Gaussian functions, and its phase front is not the familiar spherical one. Whatever it is though, we know that it matches the surface of the input mirror and therefore the surface of an identical mirror, at an identical optical path length from a reference starting point. This enables us to substitute the dark port with a signal recycling mirror, to enhance the feeble carrier fundamental mode leakage that gravitational waves are expected to generate, and be sure it resonates. This is guaranteed by the time reversal invariance of the resulting system, with our specification. The robustness of this design is due to its symmetry, which partly compensates the loss of the standard properties the separability of $l_{-}$usually ensures. For example, in most practical cases we can assume lossless optics and use the unitarity of $\mathcal{D} \mathcal{P}(\delta) \pm$ $\mathcal{B P}(\delta)$. The general output field is $\psi_{\mathrm{DP}}=\mathcal{D} \mathcal{P} \psi_{\mathrm{IN}}$ with $\psi_{\text {DP }} \neq 0$. Applying the identity

$$
\begin{aligned}
\left\langle\psi_{\mathrm{IN}} \mid \psi_{\mathrm{IN}}\right\rangle= & \left\langle\psi_{\mathrm{IN}}|\mathbb{1}| \psi_{\mathrm{IN}}\right\rangle \\
= & \left\langle\psi_{\mathrm{IN}}\right| \mathcal{B} \mathcal{P}^{\dagger} \mathcal{B P}+\mathcal{D} \mathcal{P}^{\dagger} \mathcal{B P}+\mathcal{B} \mathcal{P}^{\dagger} \mathcal{D} \mathcal{P} \\
& +\mathcal{D} \mathcal{P}^{\dagger} \mathcal{D} \mathcal{P}\left|\psi_{\mathrm{IN}}\right\rangle \\
= & \left\langle\psi_{\mathrm{IN}}\right| \mathcal{B} \mathcal{P}^{\dagger} \mathcal{B P}-\mathcal{D} \mathcal{P}^{\dagger} \mathcal{B P}-\mathcal{B} \mathcal{P}^{\dagger} \mathcal{D} \mathcal{P} \\
& +\mathcal{D} \mathcal{P}^{\dagger} \mathcal{D} \mathcal{P}\left|\psi_{\mathrm{IN}}\right\rangle
\end{aligned}
$$

and assuming the system is driven by an eigenmode of the bright port operator

$$
\mathcal{B} \mathcal{P} \psi_{\mathrm{IN}}=\lambda \psi_{\mathrm{IN}}
$$

we find that energy is conserved and the minimum amount of power is lost, when

$$
|\lambda|^{2}=1-\frac{\left|\psi_{\mathrm{DP}}\right|^{2}}{\left|\psi_{\mathrm{IN}}\right|^{2}}
$$

is an absolute maximum.

We remark this result, because the governing equation is non-Hermitian and the eigenmodes do not form an orthonormal basis, even when the optics are lossless and represented by unitary scattering matrices. For example $\left|\psi_{\mathrm{DP}}\right|^{2}$ is not equivalent to considering the individual nonresonating modes and summing the amounts of power each of them carries out of the system, because there are crossmodal terms and they can significantly add to the power $\left|\psi_{\mathrm{DP}}\right|^{2}$.

Before we specialize our optimization method and solve some specific problems, we address the robustness of our definition of dark port condition and assess its validity range. In most practical cases $\delta^{0}$ is a good approximation of the ideal solution

$$
\left.\frac{\mathrm{d}}{\mathrm{d} \delta}\left|\lambda_{1}(\delta)\right|\right|_{\delta=\delta^{0}}=\left.0 \quad \frac{\mathrm{d}}{\mathrm{d} \delta} \arg \left[\lambda_{1}(\delta)\right]\right|_{\delta=\delta^{0}}=0
$$

when any residual is $\mathcal{O}\left(\alpha^{3}\right)$. This means that the maximization of the reflected light intensity will suffice, at the same approximation order we have found for the ideal solution. We show that in fact the second requirement is normally satisfied within an error $\mathcal{O}\left(\alpha^{3}\right)$. We first note that

$$
\left\{\begin{array}{l}
\mathcal{B P}(\delta, \alpha)=\mathcal{B P}(-\delta,-\alpha) \\
\mathcal{B P}(\delta, 0)=\cos \delta \times \mathcal{B} \mathcal{P}(0,0)
\end{array}\right.
$$

always hold. Then the form of the derivative of the roundtrip phase has to be 


$$
\frac{\mathrm{d}}{\mathrm{d} \delta} \arg \left[\lambda_{1}(\delta)\right]=\alpha \times f\left(\delta^{2}\right)+\alpha^{2} \delta \times g\left(\delta^{2}\right)+\ldots
$$

where the first term is $\mathcal{O}(\alpha)$. This is made zero by the following system of approximate equations

$$
\begin{aligned}
\mathcal{B} \mathcal{P}(\delta) & =\mathbf{p} \cos \delta\left[1-i \tan \delta \frac{\mathbf{m}(\alpha)-\mathbf{m}(-\alpha)}{2}\right] \mathbf{p} \\
\mathcal{B} \mathcal{P}^{\dagger}(\delta) & =\mathbf{p}^{\dagger} \cos \delta\left[1-i \tan \delta \frac{\mathbf{m}(\alpha)-\mathbf{m}(-\alpha)}{2}\right] \mathbf{p}^{\dagger} \\
\mathcal{B} \mathcal{P}^{-1}(\delta) & =\mathbf{p}^{\dagger} \cos ^{-1} \delta\left[1+i \tan \delta \frac{\mathbf{m}(\alpha)-\mathbf{m}(-\alpha)}{2}\right] \mathbf{p}^{\dagger}
\end{aligned}
$$

derived for $\mathbf{p}$ and $\mathbf{m}(\alpha)=\mathbf{m}^{-1}(-\alpha)$ unitary. The provision on the eigenvalues

$$
\lambda^{*}(\delta,-\alpha)=\cos ^{2} \delta \lambda^{-1}(\delta, \alpha)
$$

that the above system of approximate equations implies and that is related to

$$
\mathcal{B P}^{\dagger}(\delta,-\alpha)=\cos ^{2} \delta \mathcal{B} \mathcal{P}^{-1}(\delta, \alpha)
$$

precludes the linear dependence of $\arg [\lambda(\alpha, \delta)]$ on $\alpha$.

To complete our demonstration, we need another implication of the above system of equations, that is

$$
|\lambda(\alpha, \delta)|=\cos \delta+\mathcal{O}(\alpha \delta)+\ldots
$$

which entails $\delta^{0}$ is of order $\alpha$ or smaller. At $\delta=\delta^{0}$ neglecting the round-trip phase is equivalent to relaxing the requirements of the dark port condition

$$
\left[\mathbf{M}^{-1}(\delta) \mathcal{D} \mathcal{P}(\delta) \mathbf{M}(\delta)\right]_{11} \simeq 0 \longleftarrow \frac{\mathrm{d}\left|\lambda_{1}(\delta)\right|}{\mathrm{d} \delta}=0 .
$$

Our method is robust and the approximation is $\mathcal{O}\left(\alpha^{3}\right)$ or smaller.

\section{Selection rules and special cases}

We have successfully propagated the concept of bright and dark port condition in a consistent way. For the operating point thus established, we have explored the problem of side band imbalance and the typical level of approximation our treatment tolerates, within which solutions are considered acceptable and our prescriptions robust. The side band balance depends on the properties of $\mathcal{S}^{ \pm}$. There may be selection rules for the side band duality to hold, even beyond the order of magnitude $\mathcal{O}\left(\alpha^{3}\right)$ we have found for the most general case. We now discuss some of these selection rules. Ideally $\delta^{0}$ provides a dark port condition that is mode selective and means no fundamental mode exits the dark port. The operator $\mathcal{D} \mathcal{P}\left(\delta^{0}\right)$ does not connect the fundamental mode to itself. This mathematical prescription may not be satisfied by any $\delta$ or may be satisfied by $\mathcal{D} \mathcal{P}\left(\delta^{0}\right)$ and its first $n$ odd powers. The order at which the side band balance is broken depends on that. Each case must be addressed separately. An example of a well-known transverse perturbation is mirror tilt and it will later serve to discuss a variety of issues. Most types of distortions are describable by the mismatch between the reflecting surface and the phase front of the unperturbed eigenmodes. When expanded in this basis

$$
\mathbf{m}(\alpha)=\mathbf{m}^{T}(\alpha) .
$$

Similar mathematical representations are used to study mirror displacement or profile changes. As a consequence $\mathbf{M}(\delta)=\mathbf{M}^{T-1}(\delta)$ can always be determined. This is an important mathematical provision, as the perturbative procedure for estimating the eigenvalues of a symmetric operator is analogous to the one we use for Hermitian operators. Selection rules also apply. They allow or forbid coupling between elements of selected eigenspaces of two generally symmetric commutating operators. Suppose we consider the invariance of a cylindrically symmetric system, when a rotation of its transverse degrees of freedom is applied, which is the typical case of well aligned circularly symmetric optics. A rotation of $180^{\circ}$ of the mirror is the same as switching the sign of the angle error. As for the impact on the first order variation of the bright port (determined in the unperturbed basis) this symmetry precludes diagonal terms of the perturbative operator and makes the dark port connect two optical modes, only if they belong to opposite parity eigenspaces. Within the perturbative regime $\lambda_{i}(\delta)$ are not linearly dependent on $\alpha$ and $\delta^{0}=0$ is actually an exact solution. Because of the selection rules $\mathcal{D} \mathcal{P}\left(\delta^{0}\right)$ has no diagonal term. All eigenvalues of the bright port matrix have a relative maximum at $\delta^{0}=0$. This example contains some remarkable features of odd perturbations. A crucial implication, among others, is

$$
\begin{array}{cc}
\frac{\mathrm{d} \lambda_{i}}{\mathrm{~d} \delta} & =-\sin \delta+\mathcal{O}\left(\alpha^{2} \delta\right) \longrightarrow \delta^{0}=0 \\
\Downarrow & =\mathbf{p} \frac{\mathbf{m}(\alpha)+\mathbf{m}(-\alpha)}{2} \mathbf{p} \rightarrow^{\mathbb{P}} \mathcal{B P}\left(\delta^{0}\right) \\
\mathcal{B} \mathcal{P}\left(\delta^{0}\right) & =\mathbf{m}(\alpha)-\mathbf{m}(-\alpha) \\
\mathcal{D} \mathcal{P}\left(\delta^{0}\right) & =\mathbf{p} \frac{\mathbf{m}}{2}-\mathcal{D} \mathcal{P}\left(\delta^{0}\right)
\end{array}
$$

under parity transformation $\mathbb{P}$. Since $\mathcal{B P}\left(\delta^{0}\right)$ is even and $\mathcal{D} \mathcal{P}\left(\delta^{0}\right)$ odd $\mathcal{S}^{+}=\mathcal{S}^{-}$. Within the perturbative regime, the fundamental mode component of the upper and lower side band is the same, at any order. From the physical point of view, we can interpret this result as a property of the eigenmodes with defined parity; their amplitude being odd or even is not a feature propagation can change. The fact that the location of the dark port is not relevant and the side band balance holds at any order, follows from the parity of the optical modes being conserved, independently from propagation.

A simple but more complex example of perturbation is a change in the radii of curvature and the consequences of mode dependent $\delta^{0}$ are analyzed next.

\section{Analytical and numerical applications}

Our conclusions so far, including results based on selection rules and related discussion of the side band bal- 
ance, rely on perturbation theory and therefore on its limits of applicability. We use optics misalignment again as an example to illustrate these limits, but criteria for perturbative methods to apply are general and they all involve a quantitative comparison of the strength of the perturbation to the degeneracy of the system, that is with the spacing between the eigenvalues of interest.

We describe the coupling between the fundamental and the excited mode, induced by the misalignment of the optical axis, by means of a unitary two-dimensional matrix $\mathbf{m}(\alpha)=\exp \left[-i \alpha \sigma_{1}\right]$. Other forms can be used and they are all equivalent within the second order approximation. Introducing the Pauli matrix $\sigma_{1}$ is convenient to make unitarity manifest. We compute the analytical form of the eigenvalues and eigenvectors of the bright port operator and find

$$
\begin{gathered}
\lambda_{1}=\left|\lambda_{1}\right| \exp \left[-i \arctan \sqrt{\left.\tan ^{2} \theta^{G}-\frac{\tan ^{2} \delta \tan ^{2} \alpha}{\cos ^{2} \theta^{G}}\right)}\right] \\
\lambda_{2}=\lambda_{1}^{*} \quad \psi_{1}(\delta) \simeq\left(\begin{array}{c}
1 \\
-i \sin \delta \sin \alpha /\left(2 \sin \theta^{G}\right)
\end{array}\right) \\
\psi_{2}(\delta) \simeq\left(\begin{array}{c}
i \sin \delta \sin \alpha /\left(2 \sin \theta^{G}\right) \\
1
\end{array}\right)
\end{gathered}
$$

where $\left|\lambda_{1}\right|=\left|\lambda_{2}\right|=\sqrt{\cos (\delta+\alpha) \cos (\delta-\alpha)}$.

The difference between the round-trip phases of the unperturbed eigenmodes is $2 \theta^{G}$ when $\alpha=0$ and corresponds to the Gouy phase. In perturbative situations $\delta^{0}=$ 0 is the optimal differential phase. This would identify the input laser beam $\psi_{\mathrm{IN}}=\psi_{1}\left(\delta^{0}\right)=\psi_{1}(0)$. A small amount of the excited mode light circulates at the side band frequencies; the overlap of the side band fields with the input beam is identical. The perturbative regime

$$
\delta^{\bmod } \alpha \ll \sin \theta^{G}
$$

roughly corresponds to the central part of the eigenvalue curves, plotted as a function of $\delta$ in Fig. 5. As discussed above $\left|\lambda_{i}\right|$ are the quantities of interest. By simple inspection of the analytical forms, we see that the optical re-

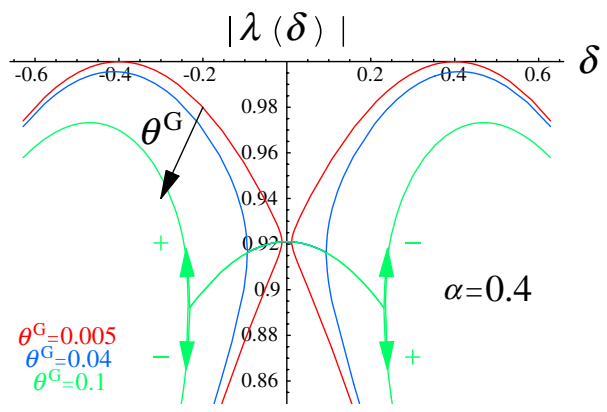

FIG. 5 (color online). The absolute value of the eigenvalues of a two-dimensional round-trip operator is shown, when the Michelson optics are misaligned and assuming three different unperturbed Gouy phases. sponse of the system varies continuously and perturbative results are reliable, within the limit $\delta \alpha \ll \sin \theta^{G}$. If $\theta^{G} \rightarrow$ 0 the problem is no longer perturbative

$$
\begin{aligned}
& \lambda_{1}=\cos (\delta+\alpha)+\mathcal{O}\left(\sin ^{2} \theta^{G}\right) \\
& \lambda_{2}=\cos (\delta-\alpha)+\mathcal{O}\left(\sin ^{2} \theta^{G}\right) \\
& 1 \\
& \psi_{1}(\delta) \simeq\left(\begin{array}{c}
\exp \left[-i \sin \theta^{G} /(\tan \delta \tan \alpha)\right]
\end{array}\right) \\
& \psi_{2}(\delta) \simeq\left(\begin{array}{c}
-\exp \left[-i \sin \theta^{G} /(\tan \delta \tan \alpha)\right] \\
1
\end{array}\right)
\end{aligned}
$$

and our analytical model is completely inadequate. The smaller $\theta^{G} \neq 0$ the smaller the angle error, beyond which perturbative treatments do not apply and alternative techniques must be explored. The two regimes are made manifest by Fig. 5 for increasing level of degeneracy. Part of the problem is due to the sophistication of the model. To illustrate this (4) where $\psi_{\mathrm{IN}} \propto \psi_{\mathrm{BP}}$ has been repeatedly solved. The size of the vector space has been increased each time and this results in a better approximation of $\left|\lambda_{i}(\delta)\right|$ at least for the lower order modes. A large number of transverse degrees of freedom is necessary, to represent both the perturbation and the mix of initial modes the perturbed ones are described by. This is even more true when $\theta^{G} \rightarrow 0$ which is why the last three curves of Fig. 6 overlap but not the ones in Fig. 7. There is a slower convergence of the analytical results when $\theta^{G} \rightarrow 0$. In all cases $\left|\lambda_{i}(\delta)\right| \leq 1$ as predicted above. This a consequence of the unitarity of $\mathcal{D P}(\delta) \pm \mathcal{B P}(\delta)$. As derived before

$$
|\lambda|^{2}=1-\frac{\left|\psi_{\mathrm{DP}}\right|^{2}}{\left|\psi_{\mathrm{IN}}\right|^{2}} \quad \text { with } \mathcal{B P} \psi_{\mathrm{IN}}=\lambda \psi_{\mathrm{IN}}
$$

for an ideal beam splitter.

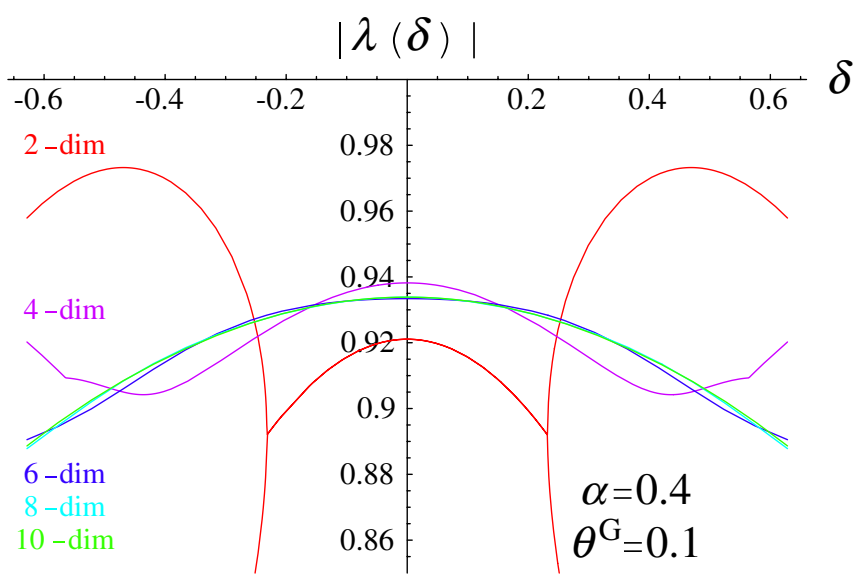

FIG. 6 (color online). The maximization of the largest eigenvalue corresponds to the optimal differential phase $\delta^{0}=0$ as selection rules impose. Related results are reliable, within perturbative limits. 


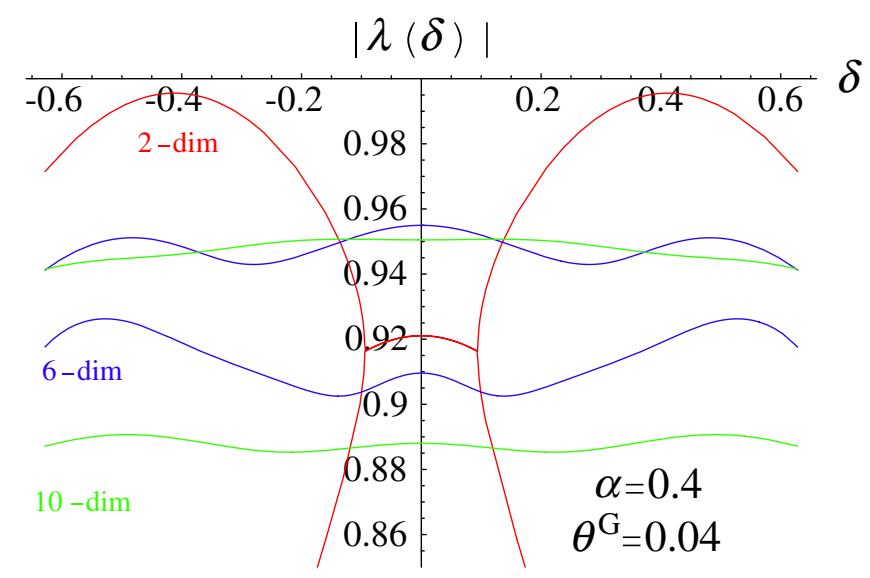

FIG. 7 (color online). The absolute value of the two largest eigenvalues is plotted and very large dissimilarities distinguish the three pairs of curves. The dependence on $\delta$ tends to become weak and the first two largest eigenvalues different. This trend is only manifest, when a larger set of unperturbed modes is used to solve the eigenvalue problem.

When $\theta^{G} \rightarrow 0$ there is no systematic hierarchy. The relevance of the lower order modes, as opposed to the negligible contribution of the higher order ones, becomes weak. The ranking itself of the eigenmodes loses its meaning. In Fig. 8 we report the absolute value of the two lowest order eigenvalues, computed by the same model, but allowing a larger unperturbed basis and increasing the size of $\mathbf{m}(\alpha)$ and $\mathbf{m}(-\alpha)$ represented. The same $\alpha=0.4$ is used. As in Fig. 6 and 7 we notice a small continuous portion around $\delta=0$ that increases with the number of unperturbed modes. When they are nearly degenerate, the system can choose from a large variety of peers and more combi-

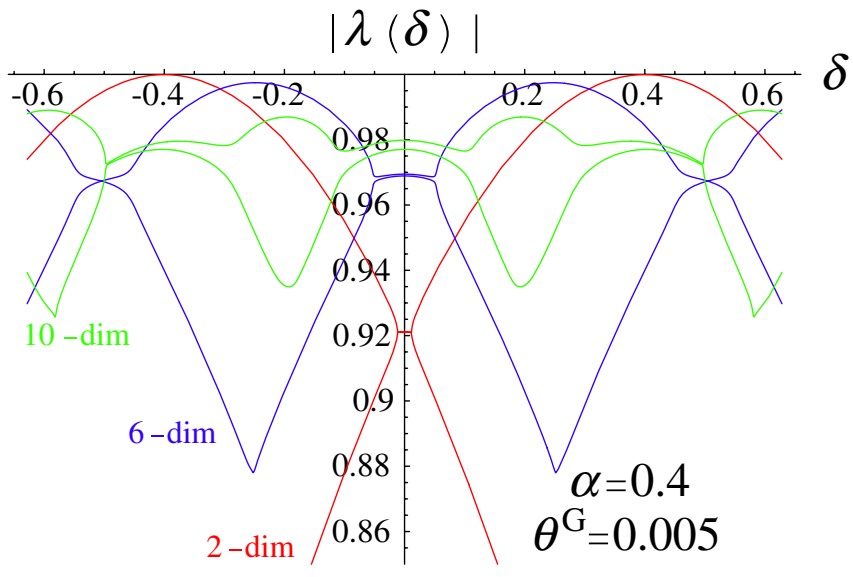

FIG. 8 (color online). This plot makes manifest the inadequacy of a small set of unperturbed modes in characterizing the behavior of the perturbed system and predicting the optimal differential phase $\delta^{0}$ and the optimal input beam. A few values correspond to maxima and they include $\delta^{0}=0$ the perturbative one. Other solutions refer to nonperturbative regimes where perturbative approximations are no longer reliable. nations can be formed, which results in many optimal input beams and differential phases.

There are a few characteristics the matric representation renders manifest. As described above, the tilted optics are related by specular symmetry and the round-trip operator $\mathcal{B P}\left(\delta^{0}=0\right)$ exhibits such property. Since this is a symmetric combination of two terms that are simply transformed into each other by spatial inversion around the tilt axis, the operator conserves parity and is considered even. Similarly the dark port operator is denoted odd; it can only connect two modes characterized by opposite parity. This not only holds for $\mathcal{D} \mathcal{P}(0)$ but for any of its odd powers. A direct consequence is that none of these sparse matrices has nonzero diagonal terms.

We devise two peculiarities:

(i) perturbative differential tilt does not change the nominal working point and this choice of the differential length satisfies the dark port condition for all perturbed modes;

(ii) the form of the dark port operator and all its odd powers makes the side band overlap with the input beam identical; this is the portion that builds up power inside the system.

For differential mirror tilt $\delta^{0}=0$ must always be one of the solutions. This comes from the first order development of the bright port operator, which implies no eigenvalue correction $\sim \delta \alpha$ around $\delta^{0}=0$. Since the first term of this expansion is an odd operator, selection rules forbid it to contribute a linear correction of the eigenvalues, as far as these refer to eigenmodes with well-defined parity. This is actually the case at $\delta^{0}=0$ but our conclusions do not extend outside an interval around it. These results are intended limited by perturbative conditions, both because the expansion of the bright port operator does not make sense otherwise and because other than at $\delta^{0}=0$ the above selection rules do not apply. This leads us to study and discuss other more general types of perturbations, that do not satisfy the selection rules of misalignment and similar. An interesting example is mirror curvature mismatch. This typical perturbation also has its own selection rules in the sense that it does not affect the dependence of the initial optical modes on the azimuthal degree of freedom. The azimuthal quantum number of the family of Laguerre-Gaussian functions therefore maintains its meaning and stays part of the mode classification. Because of its properties, this perturbation enables first order selfcoupling and linear corrections of $\left|\lambda_{i}\right|$ are now allowed. This implies a linear mode dependent correction of the optimal differential phase, that is each mode has its own. This is made manifest by the matric representation of the dark port, since the adjustment of the differential length only makes null one term along the diagonal at a time. This is in contrast to the previous case of tilt where $\mathcal{D P}\left(\delta^{0}=\right.$ 0 ) has no diagonal element. Among all $\left|\lambda_{i}\right|$ we must therefore identify the absolute maximum. This also sets $\delta^{0}$ and the form $\psi_{\mathrm{IN}}=\psi_{i}\left(\delta^{0}\right)$ that guarantees the best power build 
up. Here we can see the complexity of establishing an optimal operating point. The location of the beam splitter must satisfy the dark port condition, that is it must coincide with the dark fringe of the fundamental mode. But since $\delta$ is no longer a separable variable, any adjustment affects the modal content of the fundamental mode itself. Were the perturbation time-varying, both the position of the beam splitter and the form of the input beam should ideally follow it. The former is a practical problem standard length control techniques are able to handle; the latter does not admit a realistic implementation, as a dynamic change of the input beam is not attainable. This means that not the entire input power is efficiently used and a portion of it drives parasitic modes, which are affected by large losses at the dark port. In addition to their poor optical gain, when the system is locked on the input beam, this is the only one to have the proper round-trip phase for building up power inside the system. The efficiency of this second suppression mechanism depends on the degeneracy of the system. For spheroidal deformations $\theta^{G} \rightarrow 0$ results in a solution of (4) similar to the analytical results obtained for tilt. Again we impose $\psi_{\mathrm{IN}} \propto \psi_{\mathrm{BP}}$ for the optimization of the input. We solve a two-dimensional representation of the eigenvalue problem and use it to compute the eigenvectors

$$
\begin{aligned}
\lambda_{1} & =\cos (\delta+\alpha) \exp \left[+2 i \theta^{G} \sqrt{\frac{2}{3}}\right]+\ldots \\
\lambda_{2} & =\cos (\delta-\alpha) \exp \left[-2 i \theta^{G} \sqrt{\frac{2}{3}}\right]+\ldots \\
\psi_{1}(\delta) & \simeq\left(\begin{array}{c}
\sqrt{3}-\sqrt{2} \\
\exp \left[-i \sin 2 \theta^{G} /(\tan \delta \tan \alpha)\right]
\end{array}\right) \\
\psi_{2}(\delta) & \simeq\left(\begin{array}{c}
\exp \left[-i \sin 2 \theta^{G} /(\tan \delta \tan \alpha)\right] \\
\sqrt{2}-\sqrt{3}
\end{array}\right)
\end{aligned}
$$

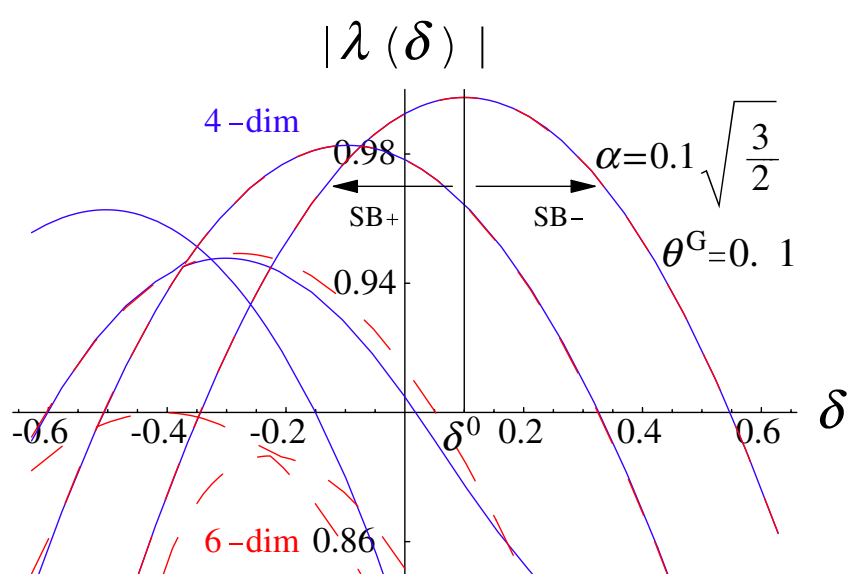

FIG. 9 (color online). The two Michelson optics are affected by opposite distortions and the maximum $|\lambda(\delta)|$ corresponds to the optimal operating point. Around $\delta^{0}$ the side bands are no longer identical. In other words, $\mathcal{S}^{+}$and $\mathcal{S}^{-}$differ by a term $\mathcal{O}\left(\alpha^{3}\right)$ as generally predicted.

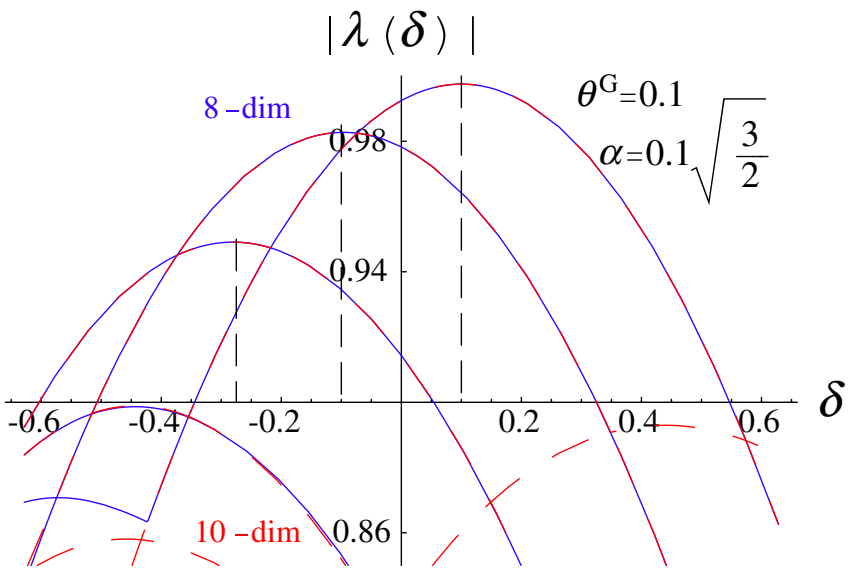

FIG. 10 (color online). Depending on the accuracy we want to achieve and the number of normal modes we want to examine, a larger number of elements of the unperturbed basis must be kept. The blue continuous line and the dashed red ones account for the $8 \times 8$ and $10 \times 10$ representation of $\mathcal{B P}(\delta)$. More of the lower order eigenvalues converge.

where $\alpha$ is a measure of the deviation from the ideal mirror profile. In this case $\alpha \propto \Delta R^{-1}$ where $R$ is the radius of curvature. A more detailed description of this particular problem is presented in Appendix B.

Here we report $\left|\lambda_{i}(\delta)\right|$ in Fig. 9 and 10. For example Fig. 9 shows overlapping curves for $i=1,2$. The size of the basis set is $D=4,6$ for the blue continuous curve and the red dashed one.

Independently from the perturbation, more sophisticated models must be used to study a nearly degenerate system, because we need a large number of transverse degrees of freedom to account for a vast variability of states and select the optimal solution among those.

When our interest is limited to the fundamental mode, perturbative corrections are reliably inferred by simple models, using small size matrices to represent the light scattering effects.

We see in Fig. 10 the stability of the fundamental mode eigenvalue. A larger basis is necessary though for estimations regarding higher order modes. This is in line with any perturbative approach and holds of course within the perturbative regime hereby discussed.

\section{E. Remarks}

We have extended the definition of dark port condition, keeping its connection with the bright port condition and identifying the fundamental mode, for which these operating conditions are established. The selected fundamental mode must be the driving beam. At this working point, the fundamental component of the circulating side bands is approximately the same. The level of the approximation corresponds to the third order or higher, using the strength of differential modal aberrations as our measure of perturbation. 
Because of the interplay of the longitudinal and transverse degrees of freedom, we find a mode dependent $\delta^{0}$ as shown in Fig. 10. Each mode has a different optical loss and the fundamental one has the lowest dark port power.

\section{F. Parasitic modes}

Since we have mentioned the negligible contribution of higher order modes, the problem is now briefly examined here, given for granted the carrier input beam resonant condition always holds. Distinguishable resonant conditions are crucial, because the optical system is similar to an open resonator, a problem where spatial boundary condi- tions make the Huygens integral a non-Hermitian operator, whose eigenmodes do not form an orthonormal set. This means that the conventional scalar product fails and the total power of a combination of modes is not the sum of the individual values [9].

The input side band field is transformed into the asymptotic circulating beam

$$
\psi_{\infty}^{ \pm} \propto \frac{1}{1-\cos \delta^{\mathrm{mod}} \mathcal{B} \mathcal{P}\left(\delta^{0}\right) \pm i \sin \delta^{\mathrm{mod}} \mathcal{D} \mathcal{P}\left(\delta^{0}\right)} \psi_{\mathrm{IN}}
$$

whose excited components are

$$
\begin{aligned}
& \frac{1}{1-\cos \delta^{\bmod } \lambda_{k}\left(\delta^{0}\right)}\left\{\mp i \sin \delta^{\bmod }\left[\mathbf{M}^{-1}\left(\delta^{0}\right) \mathcal{D} \mathcal{P}\left(\delta^{0}\right) \mathbf{M}\left(\delta^{0}\right)\right]_{k 1}-\left[\mathbf{M}^{-1}\left(\delta^{0}\right) \sin \delta^{\bmod } \mathcal{D} \mathcal{P}\left(\delta^{0}\right) \mathbf{M}\left(\delta^{0}\right)\right.\right. \\
& \left.\left.\quad \times\left(\frac{1}{1-\left.\mathbf{M}^{-1}\left(\delta^{0}\right)\left(\cos \delta^{\bmod } \mathcal{B P}\left(\delta^{0}\right) \mp i \sin \delta^{\bmod } \mathcal{D} \mathcal{P}\left(\delta^{0}\right)\right) \mathbf{M}\left(\delta^{0}\right)\right|_{\perp}}\right) \mathbf{M}^{-1}\left(\delta^{0}\right) \sin \delta^{\bmod } \mathcal{D} \mathcal{P}\left(\delta^{0}\right) \mathbf{M}\left(\delta^{0}\right)\right]_{k 1}\right\} \\
& \quad \times \frac{1}{1-\cos \delta^{\bmod } \lambda_{1}\left(\delta^{0}\right)+\mathcal{S}^{ \pm}} \psi_{\mathrm{IN}}
\end{aligned}
$$

where each $k$ is associated to one element of the higher order mode subspace. Restriction to this subspace is denoted by $\perp$ to include indices $\neq 1$. By inspection, we can see that the above formula implies an equal and opposite amplitude of the excited components of the side band fields. This holds at the first order while an imbalance of third order affects the power associated to these higher order modes.

\section{G. Standard optical models}

In our analytical model, light free propagation and interaction with an optics object are treated separately. This is a common practice and we briefly review the underlying approximations [5-7].

The basic principle is paraxial propagation, that means the collection of rays identifies a straight line. This is the longitudinal degree of freedom. The transverse distribution around it, is then represented by additional degrees of freedom according to the type of information we want to search. A unidimensional treatment enables a variety of investigations and tests, for example, to simulate the length control system and the interplay of the longitudinal degrees of freedom. Off-centered mirrors and cavity misalignment generate optical effects, that a two-dimensional model can quite predict. More complex becomes the problem and more variables must be accounted for. Even when perturbation techniques are not directly employed and numerical codes are used instead, type and size of the subset of state vectors we want to manipulate must be properly chosen and this is an issue of its own.

In fact, the convergence of the implemented routines may fail or end up with a result that is completely artificial, depending on how appropriate the setting of initial parameters is.
The number of degrees of freedom we made available for calculations is one of them. Others are responsible for initializing the correspondence between those and meaningful physical quantities.

Most numerical programs refer to a starting unperturbed configuration, that is equivalent to a basis, the action of any perturbation is computed with respect to. For example $h(\vec{r})$ identifies a surface by its deviation from a plane of reference. Reflection operators

$$
\mathcal{R}(\vec{r})=\rho(\vec{r}) \exp [-2 i k h(\vec{r})] \quad k=\frac{2 \pi \nu}{c}
$$

are then either expanded using a subset of an orthonormal family of functions or represented as two-dimensional grids. Nonuniform amplitude reflectivity is represented by $\rho(\vec{r})$ and $\rho(\vec{r})=1$ designates lossless optics.

Because of the assumption that light rays only propagate parallel to one direction, diffraction is scarcely reproduced. For example, the surface

$$
h(\vec{r})=\frac{|\vec{r}|^{2}}{2 R}+n \frac{\pi}{k} \frac{1+\operatorname{sign}(a-|\vec{r}|)}{2} \quad n \in \mathbb{N}
$$

has the same effect as the regular and smooth surface of a spherical mirror. A simple consequence of this representation of the action of reflection, is that the same set of optical modes results to be the cavity stationary fields, both for regular spherical mirrors and when these are different reflectors, as long as they are represented by the same $\mathcal{R}(\vec{r})$. Heretofore $|\vec{r}|=a$ must be a radial node of all standing wave solutions, and this is not obtained by using $\mathcal{R}(\vec{r})$ to model the reflector object. All LIGO simulation tools are based on $\mathcal{R}(\vec{r})$ and are subject to the same limitations. 
We use two of them for the numerical study of the impact of a mismatch in the curvature of the end mirrors of the recycling cavity, on the power built up by the side band fields.

We use the design parameters of the current generation of LIGO detectors; more specifically we use the configuration equivalent to geometrical match between the arms and the power recycling cavity. We do not include thermal focusing or any power dependent distortion. Therefore the power of the input beam is simply a scale factor and represents throughout our standard unit.

One of the two simulation codes we use is an objectoriented program, based on the modal approach; it relies on a finite number of Hermite-Gaussian functions and uses the subspace they identify, to construct the matrices and compute the optimal values of $\left\{l_{1}, l_{2}, L_{1}, L_{2}\right\}$ in Fig. 3. MATLAB algorithms and data structures are the substantial constituents of this model, whose name is MELODY [7]. The maximum number of independent vectors MELODY is able to allocate is 231 .

The second numerical program we use is a FORTRAN script, whose first step is a Fourier transformation of the grid representation of the optical field, then a matrix multiplication provides the paraxial propagation of the field and, when the interaction with the optics must be reproduced, the optical field momentum representation is Fourier antitransformed and each element of the field grid is multiplied by the corresponding element of $\mathcal{R}(\vec{r})$. More details and a number of applications are reported in [10] and references therein.

The FFT code and MELODY are initialized by the same setup of parameters, that is we study the response of the same optical system to the same perturbations of the ideal configuration [11].

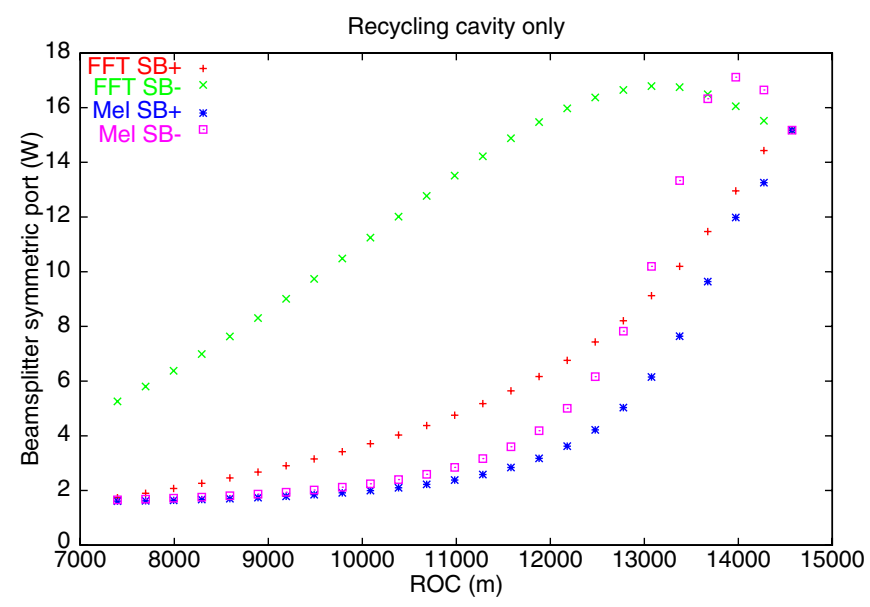

FIG. 11 (color online). Two optical simulators for LIGO detectors are used to study the power imbalance of the side bands, caused by a change of the geometry of the optical system. The ideal and unperturbed value of the radius of curvature of both end mirrors is $14571 \mathrm{~m}$. Their concave surface faces the interior of the $4 \mathrm{~km}$ long arms of the interferometer.

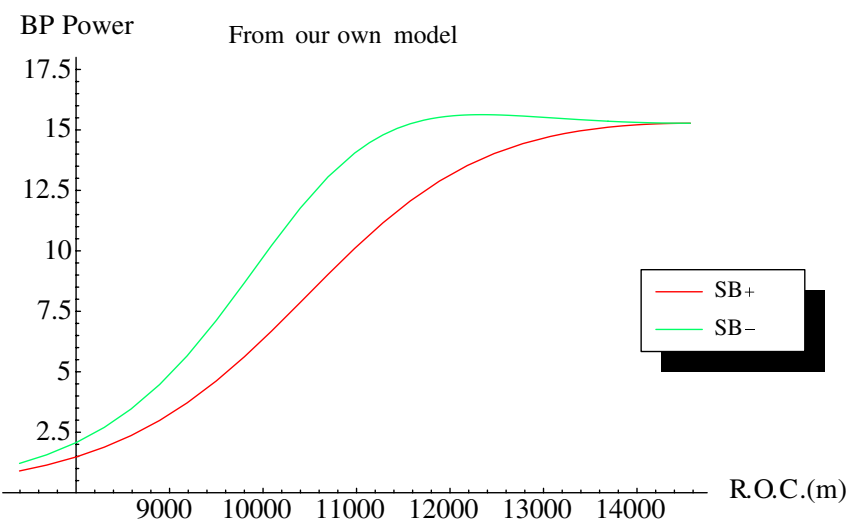

FIG. 12 (color online). We used our analytical twodimensional model and applied our specific definition of a dark port signal to compute the optimal differential length and the side band power.

For very small perturbations of the ideal optical design, we expect to find an optimal microscopic differential length (that corresponds to the most coherent reflection of an incoming electromagnetic field) for which the perturbation of the side band fields is minimized. In Fig. 11 the results of both FFT and MELODY are shown. In Fig. 12 we report our analytical results and see that the impact of the perturbation can be actually minimized by choosing the appropriate differential length.

Both our analytical model and the locking routines of the two LIGO simulators are based on the carrier, so that in all of them the side bands play no active role. Nonetheless they all use different criteria and their predictions do not overlap. This emphasizes the consequences of a nonuniversal identification of the optimal operating point.

\section{H. Resonant side bands}

Were the side bands and the carrier simultaneously resonating in the arms and the recycling cavity, their response would be distinguished by the macroscopic differential length $l_{-}$only. The arms would act as complex reflectors and a simple matrix would represent their impact on side bands and carrier. Since it would not distinguish them, our treatment so far would suffice to describe the entire interferometer as well [12].

On the contrary $L_{1}$ and $L_{2}$ are odd multiples of $c /\left(4 \nu^{\bmod }\right)$ in LIGO. The real values are actually slightly off, avoiding resonance of the secondary side bands. The result is that the side bands are very close to antiresonance and are minimally sensitive to arm length changes or other perturbations, when the carrier is resonating. The corresponding reflection is represented by a frequency dependent operator.

\section{FULL LIGO INTERFEROMETER LOCK}

We specialize our analysis of coupled resonant cavities, to the optical design of the LIGO interferometers [11]. 
Side bands and carrier have different response to variations in the arms, that means our treatment must go through a further generalization and matrices are no longer frequency independent.

The description of each Fabry-Perot arm involve the same kind of operators as $\mathbf{p}$ and $\mathbf{m}(\alpha)$. For example $\alpha=$ $k w \theta$ for mirror tilt and for curvature mismatch

$$
\alpha=\frac{k w^{2}}{2} \sqrt{\frac{3}{2}}\left(\frac{1}{R_{\text {pert }}}-\frac{1}{R_{\text {design }}}\right)
$$

where $w$ is the spot size of the beam and $k$ is the wave number. We use these perturbations as illustrative examples. In general $\alpha$ is a perturbative parameter that contains information on both the distortion and the functions we use for the expansion. This is the same set we use for describing the modal content of the beams. When the only loss of the resonator is the scalar transmittance of the input mirror, its action as complex reflector is represented by the following matrix

$$
\mathcal{R}_{\text {arm }}=\mathbf{m}(\alpha)\left(\mathbb{1}-\frac{2}{\mathbb{1}+\frac{\mathcal{F}}{\pi}\left(\mathbf{P}_{\mathbf{R T}}{ }^{-1}-\mathbb{1}\right)}\right) \quad \mathcal{F} \rightarrow \infty
$$

where $\mathbf{P}_{\mathbf{R T}}$ denotes the internal round-trip propagator. We throughout assume $\mathbf{m}(\alpha)$ and $\mathbf{P}_{\mathbf{R T}}$ unitary. Here $\mathbf{m}(\alpha)$ is the prompt reflection operator. Because of the high Finesse $\mathcal{R}_{\text {arm }}=\mathbf{m}(\alpha)$ for most frequencies. A sign flip only affects the resonating modes. For $\mathcal{F} \rightarrow \infty$ in fact $\mathcal{R}_{\text {arm }}=$ $-\mathbf{m}(\alpha)$ at resonance. Intermediate values of $\mathcal{F}$ involve intermediate results. We also find that $\mathcal{R}_{\text {arm }}$ is unitary and energy is conserved, because the only cause of optical loss is the scalar transmittance of the input mirror. Applying the general exact identity

$$
\frac{1}{\mathbb{1}+\mathcal{D}_{h}}=\frac{1}{\mathbb{1}+\mathcal{D}_{0}}-\frac{1}{\mathbb{1}+\mathcal{D}_{0}}\left(\mathcal{D}_{h}-\mathcal{D}_{0}\right) \frac{1}{\mathbb{1}+\mathcal{D}_{h}}
$$

to the expansion of

$$
\mathcal{D}_{h}=\frac{\mathcal{F}}{\pi}\left(e^{-i \phi(h)} \mathbf{P}_{\mathbf{R T}}{ }^{-1}-\mathbb{1}\right) \quad \mathcal{D}_{0}=\frac{\mathcal{F}}{\pi}\left(\mathbf{P}_{\mathbf{R T}}{ }^{-1}-\mathbb{1}\right)
$$

we find that maximum and minimum sensitivity to small scalar shifts is around resonance $\propto \mathcal{F}$ and antiresonance $\propto$ $\mathcal{F}^{-1}$. Similar expansions are useful to investigate the effect of tensorial perturbations. We will extensively apply the formula above, as it is suitable to a comprehensive treatment of the carrier and side band response to any type of deviation from ideal, in any dimension.

\section{A. Signal extraction}

Gravitational wave interferometers aim to measure the differential phase that laser light is affected by, when it enters the orthogonal arms of the detector and senses the time-varying relative displacement of the cavity optics. One of the possible causes for this effect, is a propagating space-time distortion we observe as a differential fractional change in the arm lengths, as a result of the induced space strain [13]. This phenomenon is predicted by the general theory of relativity and can have different forms; the FabryPerot optics play the role of test masses and their relative distance is affected [14]. The observable quantity is therefore the differential optical phase, caused by a traversing gravitational wave [15]. The overall effect depends on the characteristics of the astrophysical signal, including polarization and direction of propagation. To enhance the beam phase shift, the Finesse of the Fabry-Perot cavities must be very high and the input field must be a resonating mode. Because of the phase modulation of the input beam [16]

$$
\begin{aligned}
& \mathcal{R}_{\text {arm }}\left(\psi_{\mathrm{IN}} \exp \left[i \frac{2 \pi \nu^{0} t}{c}\right] \exp \left[i \Gamma \cos \frac{2 \pi \nu^{\mathrm{mod}} t}{c}\right]\right) \\
& =J_{0}(\Gamma) \mathcal{R}_{\mathrm{arm}}\left(\nu^{0}\right) \psi_{\mathrm{IN}} \exp \left[i \frac{2 \pi \nu^{0} t}{c}\right] \\
& \quad+i J_{1}(\Gamma) \mathcal{R}_{\mathrm{arm}}\left(\nu^{0}+\nu^{\mathrm{mod}}\right) \psi_{\mathrm{IN}} \exp \left(\frac{2 i \pi\left(\nu^{0}+\nu^{\mathrm{mod}}\right) t}{c}\right) \\
& \quad+i J_{1}(\Gamma) \mathcal{R}_{\mathrm{arm}}\left(\nu^{0}-\nu^{\mathrm{mod}}\right) \psi_{\mathrm{IN}} \exp \left(\frac{2 i \pi\left(\nu^{0}-\nu^{\mathrm{mod}}\right) t}{c}\right) \\
& \quad+\ldots
\end{aligned}
$$

where the frequency dependence of $\mathcal{R}_{\text {arm }}$ is made manifest. Since the system is designed to make the side bands antiresonant when the carrier beam resonates in the cavity

$$
\begin{aligned}
\mathcal{R}_{\mathrm{arm}}^{h}\left(\nu^{0}\right) & =-\mathbf{m}(\alpha)\left(1+\frac{2 i \mathcal{F}}{\pi} \phi(h)\right) \\
\mathcal{R}_{\mathrm{arm}}^{h}\left(\nu^{0} \pm \nu^{\mathrm{mod}}\right) & =\mathbf{m}(\alpha)\left(1+\left(\frac{2 i \mathcal{F}}{\pi}\right)^{-1} \phi(h)\right)
\end{aligned}
$$

and we can use the side bands, as a reference for the measurement of the phase variation of the carrier. In fact, in reflection

$$
\begin{aligned}
V_{\mathrm{arm}}\left(\nu^{\mathrm{mod}}\right) \propto & \left\langle\psi_{\mathrm{IN}}\left|\mathcal{R}_{\mathrm{arm}}^{\dagger}\left(\nu^{0}-\nu^{\mathrm{mod}}\right) \mathcal{R}_{\mathrm{arm}}\left(\nu^{0}\right)\right| \psi_{\mathrm{IN}}\right\rangle \\
& -\left\langle\psi_{\mathrm{IN}}\left|\mathcal{R}_{\mathrm{arm}}^{\dagger}\left(\nu^{0}\right) \mathcal{R}_{\mathrm{arm}}\left(\nu^{0}+\nu^{\mathrm{mod}}\right)\right| \psi_{\mathrm{IN}}\right\rangle
\end{aligned}
$$

is the oscillating observable quantity, caused by the beat of carrier and side bands, whose imaginary and real parts are extracted by in-phase and quadrature demodulation of the reflected power. When the mirror separation is changed and a length variation occurs

$$
\begin{aligned}
V_{\text {arm }}\left(\nu^{\bmod }\right)= & \left\langle\psi_{\mathrm{IN}}\right|-\mathbf{m}^{\dagger}(\alpha) \mathbf{m}(\alpha)\left(1+\frac{2 i \mathcal{F}}{\pi} \phi(h)\right) \\
& +\left(1-\frac{2 i \mathcal{F}}{\pi} \phi(h)\right) \mathbf{m}^{\dagger}(\alpha) \mathbf{m}(\alpha)\left|\psi_{\mathrm{IN}}\right\rangle \\
= & -2 \frac{2 i \mathcal{F}}{\pi} \phi(h)
\end{aligned}
$$

with $\mathbf{m}^{\dagger}(\alpha) \mathbf{m}(\alpha)=\mathbb{1}$ and $\phi(h) \simeq 0$.

A similar extraction technique is applied to the antisymmetric combination of the two reflected beams. This method is convenient, because the oscillating term of the 
output power is ideally proportional to the differential phase: the quadrature demodulation is the gravitational wave observable. This is also used to control the differential length of the two arms, while no feedback involves the in-phase demodulation. We introduce $\mathcal{R}_{\text {Mich }}$ to designate the antisymmetric combination of the arm reflections. A differential phase is included, to take into account the position of the beam splitter with respect to the arm input mirrors. For a purely differential phase $\pm \phi(h)$ from the carrier resonance

$$
\begin{aligned}
& \mathcal{R}_{\text {Mich }}^{h}\left(\nu^{0}\right)=-\mathbf{m}(\alpha) \frac{e^{i \delta^{0}}\left(1+\frac{2 i \mathcal{F}}{\pi} \phi(h)\right)-e^{-i \delta^{0}}\left(1-\frac{2 i \mathcal{F}}{\pi} \phi(h)\right)}{2} \\
& \mathcal{R}_{\text {Mich }}^{h}\left(\nu^{0} \pm \nu^{\bmod }\right)=\mathbf{m}(\alpha) \frac{e^{i\left(\delta^{0} \pm \delta_{\text {mod }}\right)}-e^{-i\left(\delta^{0} \pm \delta_{\text {mod }}\right)}}{2}
\end{aligned}
$$

In the ideal case $\delta^{0}=0$ and $\phi(h)$ is efficiently detected. In order to examine more realistic situations, we consider a complex differential phase

$$
\phi(h) \rightarrow \phi(h)+i \mathcal{L}
$$

to include differential losses.

In all LIGO detectors, this is a quite relevant effect and the observed output power is actually dominated by the constant amount of carrier light, that this type of asymmetry generates.

We will use a schematic diagram to visualize all these mechanisms and signals, although a detailed description of the length sensing system is not our focus and is described in [4].

The carrier dark port field can be represented by two orthogonal vectors that correspond to the horizontal and vertical arrows in Fig. 13. In ideal cases $V_{Q}$ is parallel to the term $\sim \phi(h)$ and $V_{I}$ to $\sim \mathcal{L}$.

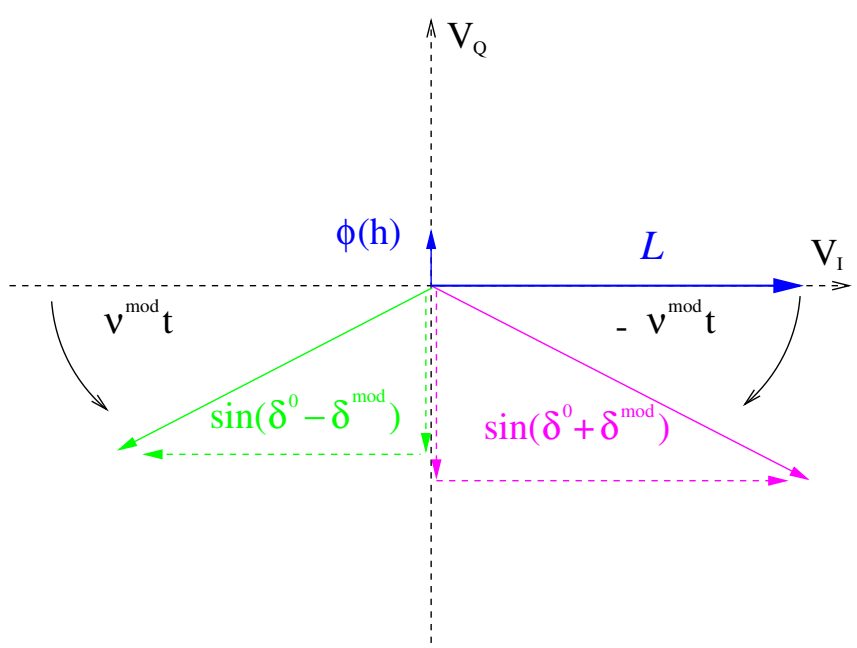

FIG. 13 (color online). The demodulation of the output power produces two orthogonal channels and one of them constitutes the gravitational wave signal $\sim \phi(h)$. These channels are orthogonal to each other, as they correspond to orthogonal phases of the demodulation function.
With our usual notations

$$
\begin{aligned}
V_{\text {Mich }}\left(\nu^{\text {mod }}\right) \propto & \left\langle\psi_{\mathrm{IN}}\left|\mathcal{R}_{\text {Mich }}^{\dagger}\left(\nu^{0}-\nu^{\text {mod }}\right) \mathcal{R}_{\text {Mich }}\left(\nu^{0}\right)\right| \psi_{\mathrm{IN}}\right\rangle \\
& -\left\langle\psi_{\mathrm{IN}}\left|\mathcal{R}_{\text {Mich }}^{\dagger}\left(\nu^{0}\right) \mathcal{R}_{\text {Mich }}\left(\nu^{0}+\nu^{\mathrm{mod}}\right)\right| \psi_{\mathrm{IN}}\right\rangle \\
= & 2\left(\sin \delta^{0}+\frac{2 \mathcal{F}}{\pi} \phi(h) \cos \delta^{0}\right) \cos \delta^{0} \sin \delta^{\mathrm{mod}} \\
& -2\left(\frac{2 i \mathcal{F}}{\pi} \mathcal{L} \cos \delta^{0}\right) \sin \delta^{0} \cos \delta^{\mathrm{mod}}
\end{aligned}
$$

is the analog of $V_{\text {arm }}\left(\nu^{\mathrm{mod}}\right)$. Because of the high $\mathcal{F}$ small differential losses can generate a significant effect; a careful tuning of the demodulation phase must be done, in order to keep the real and imaginary parts distinguished. The importance of this experimental adjustment is made manifest by the graphical example in Fig. 14. There $\theta$ plays the role of a phase rotation of the reference provided by the side bands. Typical examples are $\exp \left[ \pm 2 i \pi \nu^{\bmod }\left(2 L_{+} / c\right)\right] \neq-1$ and the same with $l_{+}$. We denote by $\theta$ any of these side band differential phases. This results in a phase shift of the oscillating observable quan-

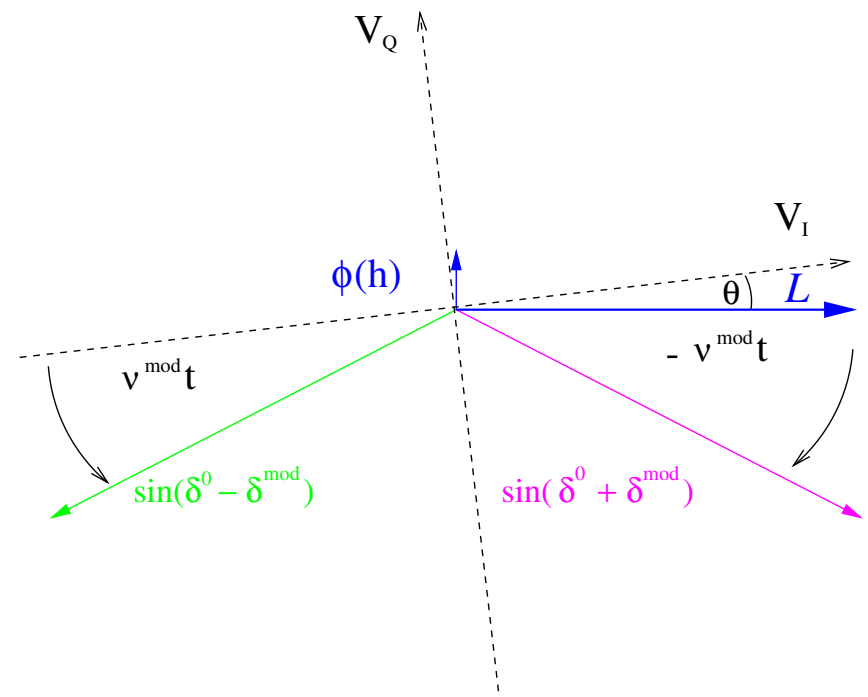

FIG. 14 (color online). The scalar product of the displayed carrier and side band vectors can be decomposed in two terms, each of which corresponds to the projection on one of two orthogonal axes. 
tities, with respect to the modulation phase at the input. This is clear from

$$
\mathcal{R}_{\text {Mich }}^{h}\left(\nu^{0} \pm \nu^{\text {mod }}\right)=e^{ \pm \imath \theta} \mathbf{m}(\alpha) \frac{e^{i\left(\delta^{0} \pm \delta_{\bmod }\right)}-e^{-i\left(\delta^{0} \pm \delta_{\text {mod }}\right)}}{2}
$$

where such a phenomenological differential shift $\exp ( \pm i \theta)$ is added. The form $V_{\text {Mich }}\left(\nu^{\text {mod }}\right)$ is proportional to $\exp (i \theta)$. Nonetheless we recover $V_{Q} \propto \phi(h)$ by simply phaserotating $V_{I}$ and $V_{Q}$. Phase tuning the demodulating sinusoidal signal is the experimental equivalent of a rotation among those signals. What we are unable to recover is a relative phase shift between the reference phase of the side bands and the reference phase of the carrier. If we consider

$$
\mathcal{R}_{\text {Mich }}^{h}\left(\nu^{0} \pm \nu^{\mathrm{mod}}\right)=e^{i \varphi} \mathbf{m}(\alpha) \frac{e^{i\left(\delta^{0} \pm \delta_{\mathrm{mod}}\right)}-e^{-i\left(\delta^{0} \pm \delta_{\mathrm{mod}}\right)}}{2}
$$

the oscillating antisymmetric port signal generated by the beat of side bands and carrier fields is

$$
\begin{aligned}
V_{\text {Mich }}\left(\nu^{\text {mod }}\right) \propto & 2 \cos \delta^{0} \sin \delta^{\bmod }(\phi(h) \cos \varphi+\mathcal{L} \sin \varphi) \frac{2 \mathcal{F}}{\pi} \\
& \times \cos \delta^{0}-2 i \sin \delta^{0} \cos \delta^{\bmod }(\mathcal{L} \cos \varphi \\
& -\phi(h) \sin \varphi) \frac{2 \mathcal{F}}{\pi} \cos \delta^{0}
\end{aligned}
$$

where we have only kept the terms $\sim \mathcal{F}$. We note that $\delta^{0}=$ 0 is equivalent to $V_{I}=0$ and $V_{Q}$ contains an unwanted term $\sim \mathcal{L}$. When some low frequency perturbation induces a differential time-dependent loss, we observe an undistinguishable mix of $\sim \mathcal{L}(t)$ and $\phi(t)$. Generally $\sin \varphi$ is an ultra-low frequency term and can be considered a constant. We refer to Fig. 15 for the graphical illustration of this mechanism.

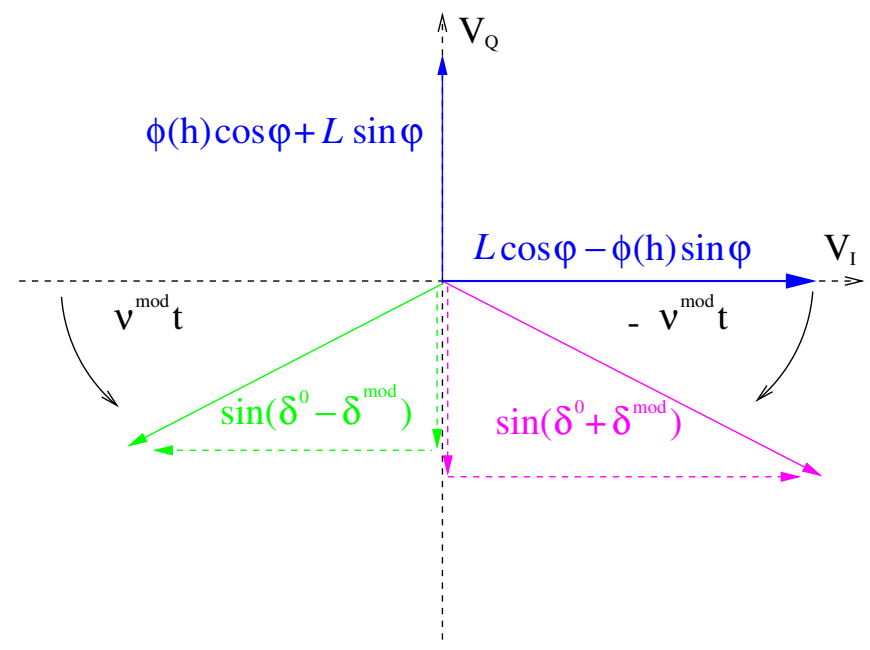

FIG. 15 (color online). No appropriate phase tuning of the demodulating sinusoidal signal can disentangle the mix of $\phi(h)$ and $\mathcal{L}$. They are coupled by $\varphi$ and this corresponds to a shift of the side band resonance point with respect to the carrier one.
Typical examples are effects related to temperature drifts; such as the change of the refractive index of the arm input optics. As the substrate is internal to the recycling cavity, it affects the eigenvalues and eigenmodes of the side band system. Because their resonant length is perturbed and the carrier one is not, the relation between the carrier and the side band phase is no longer the nominal one. A slowly varying $\varphi$ can therefore be the explanation for a rotation, that is observed between the in-phase and quadrature components of a few control signals, when the full interferometer is locked and a large amount of power circulates in the arms, causing an increase in the temperature of the test mass mirrors. The effects of this phenomenon are partially neutralized by the thermal compensation system.

In the next subsection we will focus on tensorial perturbations and apply the expansion of multidimensional $\mathcal{R}_{\text {arm }}(\nu)$ to transverse distortions. Both common and differential effects will be considered, because the frequency dependent arm reflection makes their impact on the side bands and the carrier distinguishable, with interesting consequences.

From the mathematical point of view, the eigenvalues and the optimal operating point of the full interferometer are shifted by frequency dependent amounts, a phenomenon that modifies the relation between the reference phase of the side bands and the carrier.

\section{B. Frequency dependent modal aberrations}

Side bands and carrier are distinguished by both $l_{-}$and arm reflection. The result is that the equivalent of $\mathbf{m}(\alpha)$ is frequency dependent. The unidimensional treatment already contains the ingredients for a discussion on side band imbalance and its impact on the detector error signals; phase shifts are the phenomenological effect of such a frequency dependence, as carrier and side bands respond differently to perturbations of the arm object. Our further analysis regards the multidimensional aspect. As in Sec. III we assume the common configuration as our unperturbed standard; because of the frequency dependent arm reflection, we must also assign its microscopic length and refer all perturbations to that.

There is an important point worth noting; unless the unperturbed optical modes are purely Hermite-Gaussian or Laguerre-Gaussian functions, the profile of the arm input mirror does not match the phase front of all modes; that is there is no unique septum in Fig. 2 working for all modes. The equivalence of the phase fronts is not a general ubiquitous property; it holds for stationary modes in coincidence of the boundary. In the general case $\mathbf{m}(\alpha)$ is factored out. We assume

$$
[\mathbf{m}(\alpha)]_{11}=1+\mathcal{O}\left(\alpha^{2}\right) \quad\left[\mathbf{P}_{\mathbf{R T}}^{0}\right]_{11}=1
$$

where $\mathbf{P}_{\mathbf{R T}}^{\mathbf{0}}=\mathbf{m}(\alpha) \mathbf{P}_{\mathbf{R T}}$. This is made possible by 


$$
\mathbf{m}^{\dagger}(\alpha)=\mathbf{m}(-\alpha) \longrightarrow[\mathbf{m}(\alpha)-\mathbb{1}]_{11} \propto i \alpha
$$

which implies a mode selective microscopic adjustment of the arm input mirror by a displacement that is also appropriate for the other side reflection. Here $\mathbf{m}(\alpha)$ and $\mathbf{m}^{\dagger}(\alpha)$ refer to the two mirror sides [5-7].

We apply this formalism to the expansion of the carrier arm reflection matrix

$$
\begin{aligned}
\mathcal{R}_{\text {arm }}\left(\nu^{0}\right)= & \mathbf{m}(\alpha)\left[\mathbb{1}-\frac{2}{\mathbb{1}+\frac{\mathcal{F}}{\pi}\left(\mathbf{P}_{\mathbf{R T}}^{\mathbf{0}}{ }^{-1}-\mathbb{1}\right)}\left(\mathbb{1}-\frac{\mathcal{F}}{\pi} \mathbf{P}_{\mathbf{R T}}^{\mathbf{0}}{ }^{-1}\right.\right. \\
& \left.\left.\times(\mathbf{m}(\alpha)-1) \frac{1}{\mathbb{1}+\frac{\mathcal{F}}{\pi}\left(\mathbf{P}_{\mathbf{R T}}{ }^{-1}-\mathbb{1}\right)}\right)\right]
\end{aligned}
$$

at first order in $\alpha$. Denoting $\left[\mathbf{P}_{\mathbf{R} \mathbf{T}}^{\mathbf{0}}\right]_{j j}=\exp \left[-2 i \eta_{j}\right]$

$$
\mathcal{R}_{\text {arm }}\left(\nu^{0}\right)=\left(\begin{array}{cc}
-1 & \frac{[\mathbf{m}(\alpha)]_{1 j}}{i \tan \eta_{j}} \\
\frac{[\mathbf{m}(\alpha)]_{k 1}}{i \tan \eta_{k}} & {[\mathbf{m}(\alpha)]_{k j}}
\end{array}\right)
$$

where $[\mathbf{m}(\alpha)]_{j j}=1+\mathcal{O}(\alpha)$ for $j \neq 1$. The side bands are assumed to be so far from resonance, that the round-trip propagator has no impact on them. Therefore,

$$
\mathcal{R}_{\text {arm }}\left(\nu^{0} \pm \nu^{\text {mod }}\right)=\mathbf{m}(\alpha) .
$$

In particular, they are completely insensitive to distortions or perturbations of the end mirror.

If we expand the reflection operator in terms of the end mirror object matrix

$$
\begin{aligned}
\mathcal{R}_{\mathrm{arm}}\left(\nu^{0}\right)= & \mathbb{1}-\frac{2}{\mathbb{1}+\frac{\mathcal{F}}{\pi}\left(\mathbf{P}_{\mathbf{R T}}^{\mathbf{0}}{ }^{-1}-\mathbb{1}\right)}\left(\mathbb{1}-\frac{\mathcal{F}}{\pi} \mathbf{P}_{\mathbf{R T}}^{\mathbf{0}}{ }^{-\frac{1}{2}}\right. \\
& \left.\times(\overline{\mathbf{m}}(\bar{\alpha})-1) \mathbf{P}_{\mathbf{R T}}^{\mathbf{0}}{ }^{-\frac{1}{2}} \frac{1}{\mathbb{1}+\frac{\mathcal{F}}{\pi}\left(\mathbf{P}_{\mathbf{R T}}{ }^{-1}-\mathbb{1}\right)}\right)
\end{aligned}
$$

where we have separated

$$
\overline{\mathbf{m}}(\bar{\alpha})=\mathbf{P}_{\mathbf{R}}^{\mathbf{0}}{ }^{\frac{1}{2}} \mathbf{P}_{\mathbf{R T}}{ }^{-1} \mathbf{P}_{\mathbf{R}}^{\mathbf{0}}{ }^{\frac{1}{2}} \quad \mathbf{m}(\alpha)=\mathbf{m}(0)=\mathbb{1}
$$

so that $\mathcal{R}_{\text {arm }}\left(\nu^{0} \pm \nu^{\text {mod }}\right)=\mathbb{1}$ in this case. Under the same microscopic conditions we have above described, that allows us to keep the unperturbed fundamental mode resonant

$$
\mathcal{R}_{\mathrm{arm}}\left(\nu^{0}\right)=\left(\begin{array}{cc}
-1 & \frac{[\overline{\mathbf{m}}(\bar{\alpha})]_{1 j}}{i \sin \eta_{j}} \\
\frac{[\overline{\mathbf{m}}(\bar{\alpha})]_{k 1}}{i \sin \eta_{k}} & \mathbb{1}
\end{array}\right)
$$

within the first order approximation. For symmetric $\overline{\mathbf{m}}$ the coupling terms are real and $\mathcal{R}_{\text {arm }}\left(\nu^{0}\right)$ is also symmetric. For misalignment

$$
[\overline{\mathbf{m}}(\bar{\alpha})]_{12}=[\overline{\mathbf{m}}(\bar{\alpha})]_{21}=-i \bar{\alpha}
$$

in the appropriate basis [5]. Here $\bar{\alpha} \in \mathbb{R}$ is proportional to the angle error. One mode is excited and this is referred to by the index 2 in a Hermite-Gaussian basis.

The frequency dependence of these operators enables the construction of error signals $\propto \bar{\alpha}$ that correspond to an oscillating observable. They are analogous to $V_{\text {arm }}\left(\nu^{\bmod }\right)$ for the measurement of resonance offset. The main difference is that resonance offset is a perturbation represented by $\mathbb{1}$ so that no matrix is necessary to reveal the information. Instead a connecting tensor $\mathbf{D}$ must be inserted in the adjunct product, to make the unperturbed field beat against the excited one, as determined by the modal coupling of the perturbation

$$
\mathbf{D}_{k j}=\delta_{k 1} \delta_{j e}=\mathbf{D}_{j k}
$$

where $e$ denotes the excited mode. This form enables us to extract a quantity $\propto \bar{\alpha}$ therefore suggesting a physical observable we can measure. Typical angle error signals are constructed according to this principle. There are segmented photodetectors which operate as the connecting tensor $\mathbf{D}$. Their spatial modules are geometrically designed to extract cross-modal terms and can be connected in such a way as to provide the combinations of interest [5].

Two remarkable phenomena are made obvious by our compact formalism. One is the different sensitivity to common and differential mirror tilt, in a nearly flat and a nearly concentric optical resonator. The former situation corresponds to

$$
\frac{[\mathbf{m}(\alpha)]_{12}}{i \tan \eta_{2}}+\frac{[\overline{\mathbf{m}}(\bar{\alpha})]_{12}}{i \sin \eta_{2}} \simeq-\left(\frac{\alpha}{\tan \eta_{2}}+\frac{\bar{\alpha}}{\sin \eta_{2}}\right) \quad \eta_{2} \ll 1
$$

which makes the severity of the common effect $\alpha=\bar{\alpha}$ manifest. If we consider the equivalent nearly concentric cavity (which is characterized by the same and opposite spacing between the resonant frequencies of the optical system) the following formula results from the conversion:

$$
\frac{[\mathbf{m}(\alpha)]_{12}}{i \tan \left(\pi-\eta_{2}\right)}+\frac{[\overline{\mathbf{m}}(\bar{\alpha})]_{12}}{i \sin \left(\pi-\eta_{2}\right)} \simeq\left(\frac{\alpha}{\tan \eta_{2}}-\frac{\bar{\alpha}}{\sin \eta_{2}}\right) .
$$

The relative significance of common and differential misalignment is inverted [17].

Parametric instabilities are caused by the excitation of a resonating mode. In the subspace of the two involved optical modes, the action of the perturbation is formally described by

$$
\mathcal{R}_{\text {arm }}\left(\nu^{0}\right)=\left(\begin{array}{cc}
-1 & \frac{2 \mathcal{F}}{\pi}[\mathbf{m}(\alpha)]_{12} \\
\frac{2 \mathcal{F}}{\pi}[\mathbf{m}(\alpha)]_{21} & -1
\end{array}\right)
$$

when $\mathbf{P}_{\mathbf{R T}}^{\mathbf{0}}=\mathbf{m}(\alpha) \mathbf{P}_{\mathbf{R T}}$. We can similarly evaluate

$$
\mathcal{R}_{\text {arm }}\left(\nu^{0}\right)=\left(\begin{array}{cc}
-1 & \pm \frac{2 \mathcal{F}}{\pi}[\overline{\mathbf{m}}(\bar{\alpha})]_{12} \\
\pm \frac{2 \mathcal{F}}{\pi}[\overline{\mathbf{m}}(\bar{\alpha})]_{21} & -1
\end{array}\right)
$$

where $\overline{\mathbf{m}}(\bar{\alpha})$ represents modal scattering at the end mirror. Here $\left[\mathbf{P}_{\mathbf{R}}^{\mathbf{0}} \mathbf{T}_{22}^{\frac{1}{2}}\right]_{2}= \pm\left[\mathbf{P}_{\mathbf{R} \mathbf{T}^{\frac{1}{2}}}\right]_{11}$.

The second interesting property regards the characteristic polynomial. Because of the frequency dependence of the arm reflection, unless the perturbation has a parametric instability effect, 


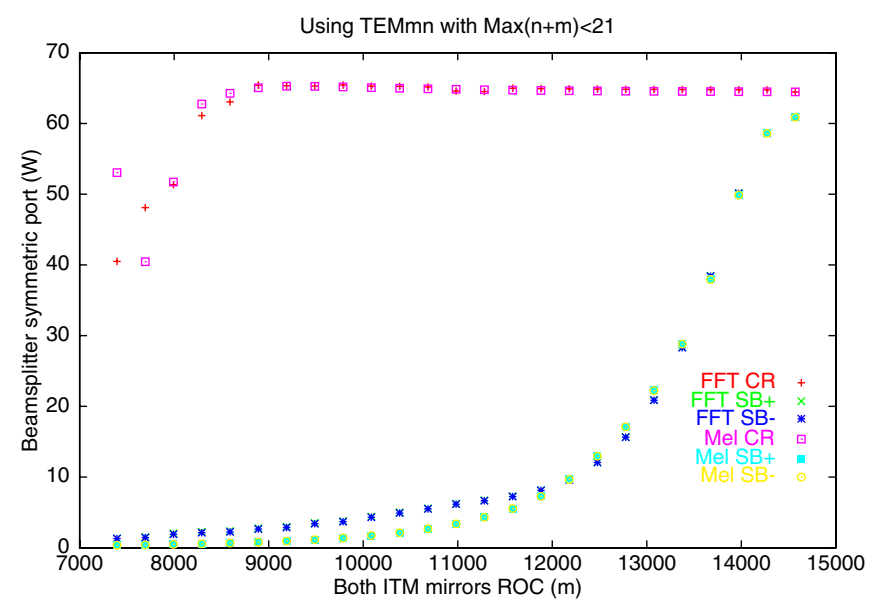

FIG. 16 (color online). The optical system the Fabry-Perot arms and the recycling cavity form is mathematically equivalent to a resonator, whose mechanical geometry depends on the probe frequency.

$$
\begin{aligned}
\left.\operatorname{det}\left[\mathcal{R}_{\operatorname{arm}}\left(\nu^{0}\right)-\lambda \mathbb{1}\right]\right|_{\lambda \simeq-1} \simeq & \frac{-2(1+\lambda)+\mathcal{O}\left(\alpha^{2}\right)}{2} \\
& \times \operatorname{det}[(1-\lambda) \mathbb{1}]
\end{aligned}
$$

that is the first eigenvalue is not affected by $\sim \alpha$ corrections. This stability of the first eigenvalue is a general result that only relies on the resonant condition of the FabryPerot cavity. Such a purely analytical feature can actually inform the optical design of the power recycling cavity; whenever this is separately degenerate, the over coupled Fabry-Perot resonator makes it antidegenerate from the point of view of the carrier and the resonant length remains robust against perturbations. In Fig. 2 this is the case for $l_{-} \ll ? ? ?_{+}$that is the LIGO design.

The stability of the carrier is contrasted by the behavior of the side bands, which become maximally sensitive. This

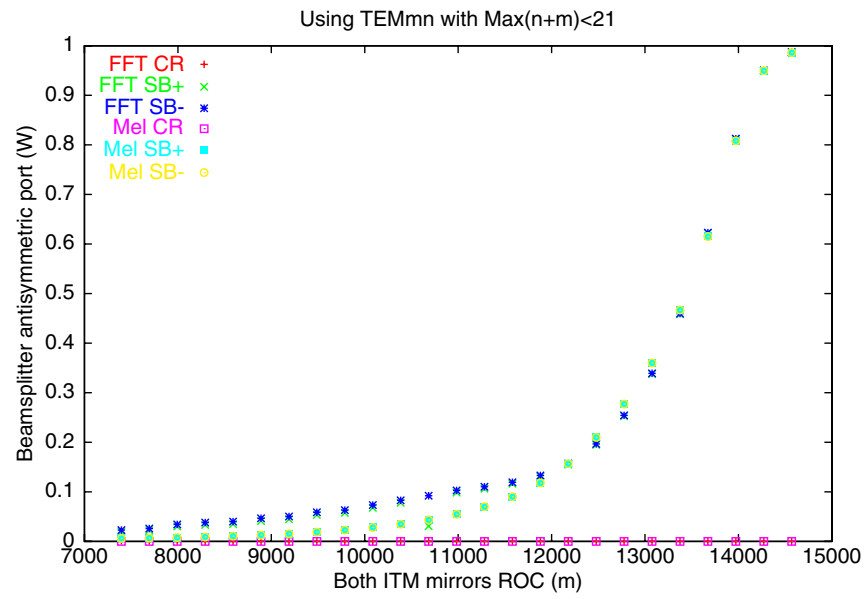

FIG. 17 (color online). The side band dark port power is proportional to the recycling cavity gain and the decrease we observe is caused by a poor side band power build up. remarkable distinction is shown in Fig. 16. Two simulation codes are used, whose numerical results coincide for identical changes of the radius of curvature $\Delta R \leq 1 \mathrm{~km}$ in both Fabry-Perot input mirrors. Both the FFT code and MELODy apply microscopic adjustments of the lengths, according to the carrier resonant condition only. This does not coincide with side band optical resonance. The drop in the power they build up is also visible in Fig. 17 showing the dark port power.

This is a manifestation of the frequency dependent arm reflection. Only if the optical match between the cavities is perfect does the carrier resonance coincide with the side band resonance. When that is spoiled by shape distortions
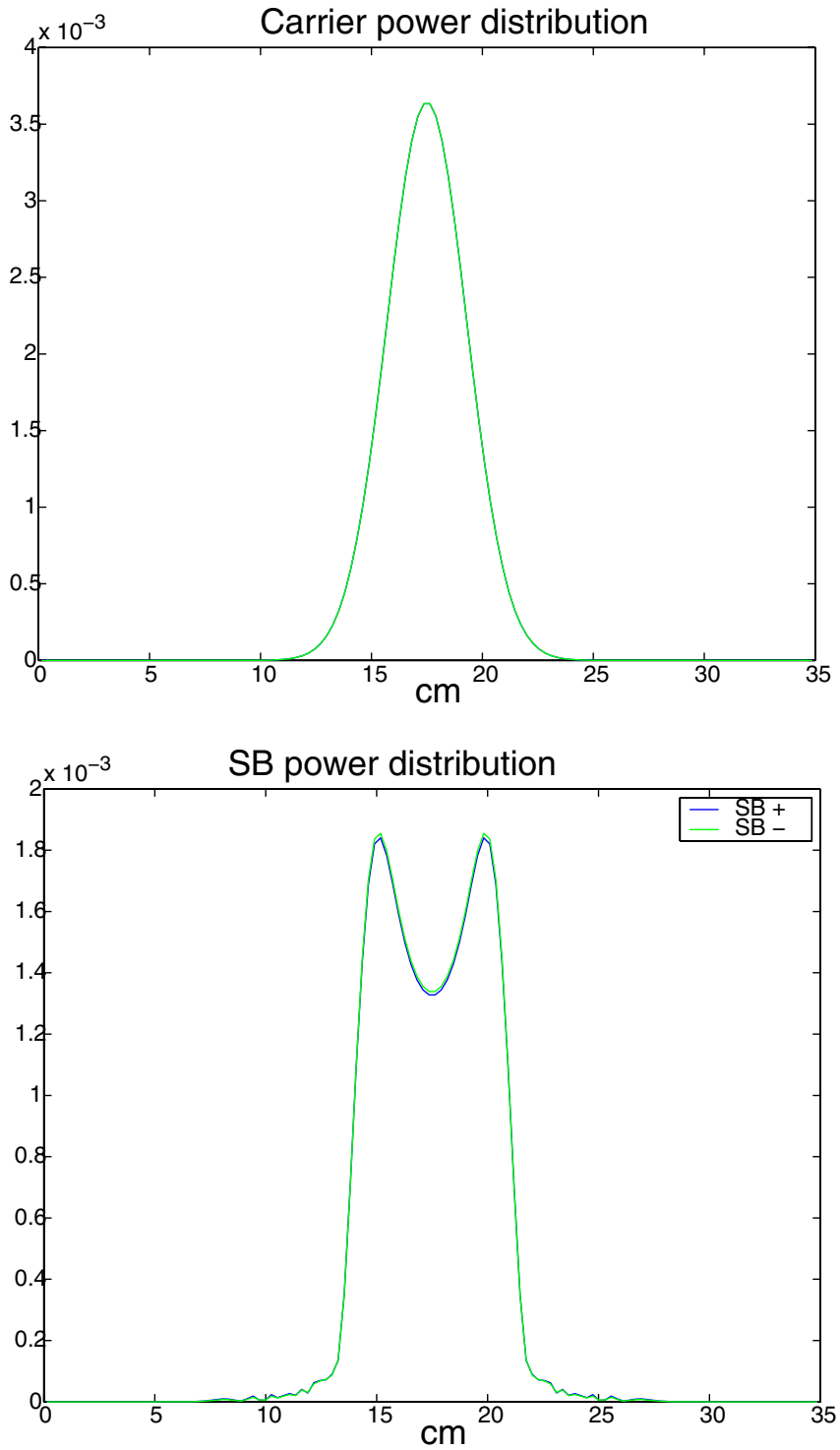

FIG. 18 (color online). The power distribution of the carrier and side band asymptotic fields is shown. The range of the horizontal axis is $35 \mathrm{~cm}$ and the distributions are normalized. These data correspond to the LIGO mismatched configuration that is for low input power. 
of the Fabry-Perot input optics, a frequency dependent variation of the resonances of the recycling cavity occurs.

\section{Common modal aberrations}

We have established the different character of the optical system, carrier and side bands relate to, as a result of the frequency dependence of the action of transverse perturbations. In Fig. 18 the frequency dependent shape of the field the recycling cavity supports is shown. The power radial profile has the expected Gaussian form and a peculiar ringshaped look, at the carrier and side band frequency, respectively.

From the point of view of the recycling cavity, the side band input field is a superposition of optical modes, whose resonances are affected by the curvature of the Fabry-Perot input optics. By adjusting $l_{+}$a different combination is formed, because the relative weight of the LaguerreGaussian constituents is a function of the longitudinal microscopic condition. The following longitudinal offsets have been applied

$$
\delta l_{+}=[-4 n m,-2 n m,+2 n m,+4 n m]
$$

with respect to the carrier resonant condition the simulation code determines. The corresponding plots of the side band power profile are shown, clockwise in Fig. 19 starting from the upper left panel. Our results demonstrate a chaotic behavior due to the inseparability of the boundary conditions, that is the longitudinal and the transverse degrees of freedom are not disjoint, so that the transverse mode indices lose their meaning. A dedicated experiment that was spurred by this characterization of the side band behavior is described in a forthcoming paper [18]. The LIGO Livingston Observatory was used to study the spatial response of the side band power distribution to microscopic changes of $l_{+}$. The measurements agree with the predictions [19].
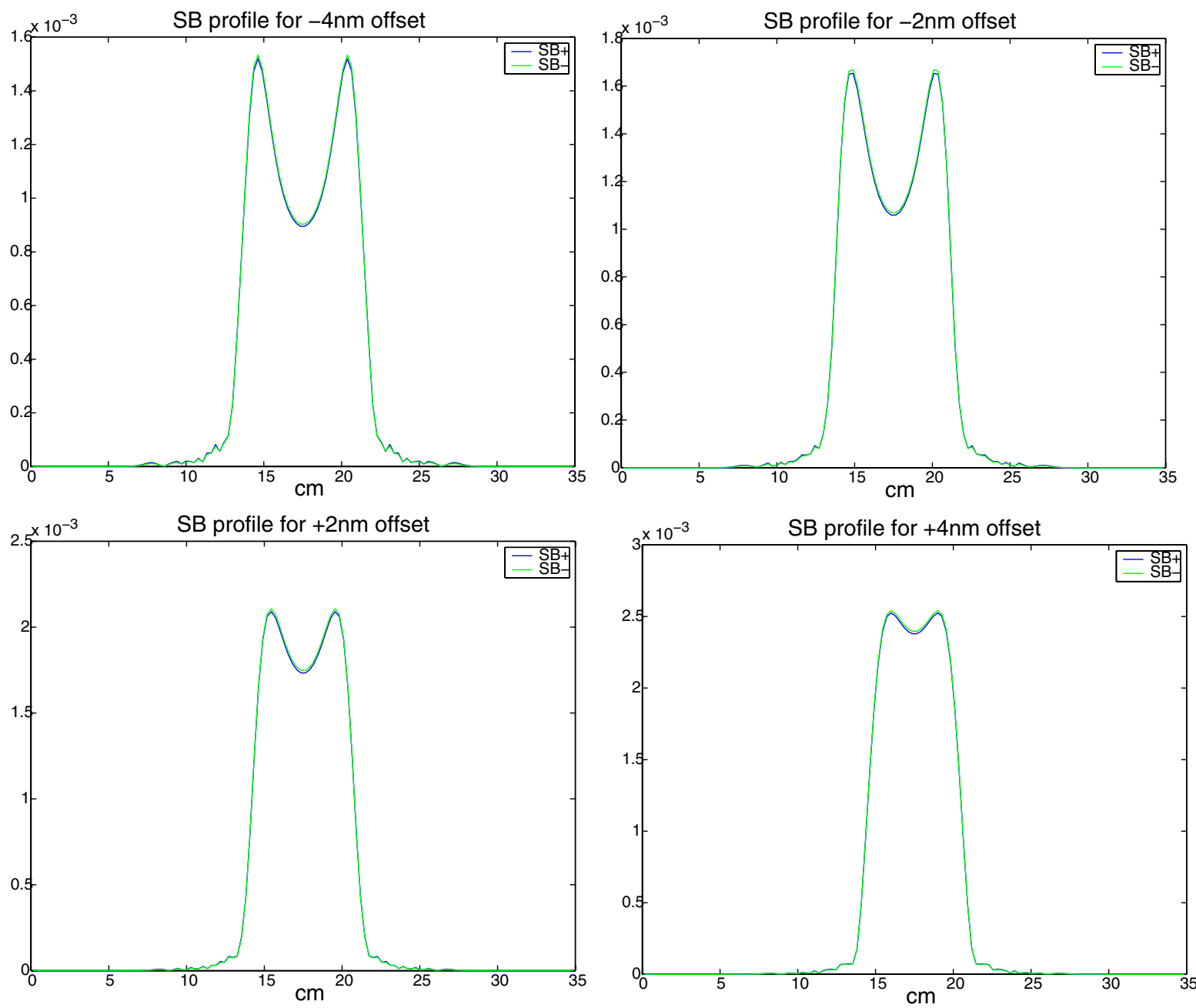

FIG. 19 (color online). The asymptotic side band field is affected by small variations of the recycling cavity length $l_{+}$. The excitation of higher order modes depends on the optical mismatch between the arms and the recycling cavity, a perturbation that is nominally zero in coincidence of the design input power and that does not affect the carrier. Power distributions are normalized. They are reported for both side bands over a total horizontal range of $35 \mathrm{~cm}$. 
A tremendous amount of work was put in the detailed comparison of the data we recorded and the mathematical predictions, especially because the length servo had to be characterized first, in order to calibrate the artificial offset the control loop was modified by, in terms of the relative distance the arm cavity input optics and the recycling mirror are separated by. This was made possible by Andri Gretarsson, Valera Frolov and Brian O'Reilly and the results of their investigation represent an important breakthrough in our understanding of the complex coupled system of which the LIGO interferometers consist. They confirmed the larger spatial magnitude of the side band field, with respect to the spot size of the input beam [20].

Previous studies had alluded to a smaller transverse distribution of the side band light, so that the experimental observations also validated the numerical simulations of one of us (E. D'A.). This investigation of the beam shape of the carrier and side band fields was instrumental to the optical characterization of the low power LIGO interferometers, that is in the study of effects induced by the most likely perturbations, when they are combined with a severe optical mismatch between the cavities [21]. In fact the LIGO detectors are designed to be a perfectly coupled system when the input power is $6 \mathrm{~W}$ and the recycling cavity is not matched otherwise. The poor optical coupling is responsible for the side band shape being remarkably different from the Gaussian input beam the unperturbed Fabry-Perot resonators are designed to support [22]. The initiative of probing such a difference was mainly due to Daniel Sigg and Joe Betzwieser and involved a variety of preliminary analyses; the accuracy of their investigations enabled the ultimate verification of the side band shape and its transverse magnitude [23].

\section{Differential modal aberrations}

We now address the analogous problem of Eq. (4). The arm cavities are carrier locked on the unperturbed fundamental mode the ideal common resonator identifies. For the sake of simplicity, $\mathbf{p}=\mathbb{1}$ as an approximation of the short optical length light traverses. In LIGO $l_{+}<10^{-3} L_{+}$ and the arm cavities are characterized by a Gouy phase $\eta_{2} \simeq 1.24$ when $\eta_{1}=0$. We conventionally set $\eta_{1}=0$ to denote the arm carrier resonant condition. We also assume that the recycling mirror matches the unperturbed input optics of the two arms. For $\mathbf{p}=\mathbb{1}$ this means it has their same surface profile. Because of the substrate of the input optics, their effect should also be included and the corresponding lens accounted for. This process is only straightforward when the lens is neither frequency dependent nor modal dependent. We will not enter these issues, but an example of the former case is thermal focusing and of the latter non-Gaussian beams. Such problems can be treated as common modal aberrations since they introduce a geometrical distinction that regards the fundamental mode, as defined by the frequency dependent common configura- tion. This distinction is only relevant to the side band problem, when coupled to an asymmetry of the interferometer. For pure differential perturbations

$$
\mathcal{B} \mathcal{P}(\delta) \simeq\left(\begin{array}{cc}
-\cos \delta+\mathcal{O}\left(\alpha^{2}\right) & \sin \delta\left(\frac{[\mathbf{m}(-\alpha)-\mathbf{m}(\alpha)]_{1_{j}}}{2 \tan \eta_{j}}\right. \\
\sin \delta \frac{[\mathbf{m}(-\alpha)-\mathbf{m}(\alpha)]_{k 1}}{2 \tan \eta_{k}} & \mathbb{1} \cos \delta+\mathcal{O}(\alpha)
\end{array}\right)
$$

satisfies the general property

$$
\begin{aligned}
\left.\frac{\operatorname{det}[\mathcal{B P}(\delta)-\lambda \mathbb{1}]}{\operatorname{det}[(\cos \delta-\lambda) \mathbb{1}]}\right|_{\lambda \alpha-\cos \delta} & -2 \cos \delta(\cos \delta+\lambda) \\
& +\mathcal{O}\left(\alpha^{2}\right)
\end{aligned}
$$

where the terms $\mathcal{O}\left(\alpha^{2}\right)$ are even functions of $\delta$. The value $\delta^{0}=0$ is the best operational point. As we assume the input beam is the unperturbed arm cavity fundamental mode, the optical coupling corresponds to a power loss $\mathcal{O}\left(\alpha^{4}\right)$ determined by $\mathcal{B P}(0)$. The perturbative approach takes advantage of the largest possible phase spacing and the entire set of higher order modes is separated from the fundamental one, which also drives the system. Were $\mathbf{p} \neq$ $\mathbb{1}$ factors $\sim \cos ^{-1} \theta_{k}^{G}$ would magnify the impact of the excitation involving the mode labeled by $k$.

\section{E. Optimal differential length}

The system being degenerate enables a few useful simplifications. In fact $\mathcal{R}_{\text {arm }}\left(\nu^{0}\right)$ has none of the symmetries of $\mathbf{m}(\alpha)$. Those were the basis for most of our considerations of Sec. III. For general $\mathcal{R}_{\text {arm }}\left(\nu^{0}\right)$ the transpose is something different, for example. Also the reversion $\alpha \rightarrow-\alpha$ does not coincide with its inversion.

Nonetheless, for antidegenerate recycling cavity the following identity holds

$$
\mathcal{B P}^{\dagger}(\delta,-\alpha)=\cos ^{2} \delta \mathcal{B P} \mathcal{P}^{-1}(\delta, \alpha)+\mathcal{O}\left(\alpha^{2}\right)
$$

from which we derive

$$
\frac{\mathrm{d}}{\mathrm{d} \delta} \arg [\lambda(\delta)] \propto \alpha^{2} \delta
$$

and $|\lambda(\alpha, \delta)|=\cos \delta+\mathcal{O}(\alpha \delta)$. This first order expansion is analogous to that of Sec. III. We find $\delta^{0}=0$ because of the relation

$$
\begin{aligned}
\mathcal{B P}^{2}(\delta)-\cos ^{2} \delta \mathbb{1}= & \left(\begin{array}{lc}
0 & 0 \\
0 & -i \sin \delta[\mathbf{m}(\alpha)-\mathbf{m}(-\alpha)]
\end{array}\right) \\
& +\ldots
\end{aligned}
$$

which precludes linear corrections $\sim \delta \alpha$ of the first eigenvalue. This means that $\delta^{0}=0$ keeps on being the carrier optimal working point.

\section{F. Nondegenerate power recycling cavity}

The characteristic behavior of the antidegenerate recycling cavity, from the point of view of the stability of the carrier field, holds over a perturbative range of parameters. This is limited by similar criteria to the ones discussed in Sec. III for $\mathbf{p} \neq \mathbb{1}$ so we review and extend those. 
As a result of the mirror distortions, the transverse and longitudinal degrees of freedom are coupled. In particular, we have seen that there is a preferred dark port location and a definition of dark port condition, which reproduce the ideal working point and the role itself of $l_{+}$and $l_{-}$as assigned by the unidimensional analysis. This propagation of the ideal working point, including simultaneous conditions and independency of the adjustments they imply, adapt to one mode the concepts of the unidimensional treatment.

These include matching the input field to the fundamental mode of the optical system, moving the beam splitter in order to minimize the carrier output power and locking $l_{+}$ on its resonance. The really novel point is that $l_{-}$plays a role in all these. Being no longer a separable variable $l_{-}$ determines the antisymmetric port loss but also the eigenmodes and eigenvalues of the system.

This dependence and the interrelation of the microscopic length conditions is a small violation, when

$$
\delta \alpha \ll \sin \theta^{G} \quad \delta \propto l_{-}
$$

that is within a perturbative regime. As a consequence $\theta^{G}$ ought not be small; this quantity refers to the phase spacing between the resonances of the recycling cavity. On the contrary $\theta^{G} \rightarrow 0$ is the ideal match of a single mode resonator. Similar considerations to those of Sec. III apply to coupled cavities. For the carrier field

$$
\delta \times \frac{[\mathbf{m}(-\alpha)-\mathbf{m}(\alpha)]_{1 j}}{2 i \tan \eta_{j}} \ll \cos \theta_{j}^{G}
$$

is the perturbative regime.

The frequency dependence of the perturbation results in a frequency dependent characterization of the field stability. In particular $l_{-}$and $l_{+}$require frequency dependent adjustments and this leads to consequences that must also be considered perturbative effects.

From the two above semiquantitative assessments, reflecting the properties of degenerate resonators and antidegenerate coupled systems, we derive the limit

$$
\begin{aligned}
\delta\left|\frac{\mathbf{m}(\alpha)-\mathbf{m}(-\alpha)}{2}\right| & \ll \sin \theta_{j}^{G} \\
& \ll \sqrt{1-\delta^{2}\left|\frac{\mathbf{m}(\alpha)-\mathbf{m}(-\alpha)}{2 \tan \eta_{j}}\right|^{2}}
\end{aligned}
$$

within which the contribution of the excited modes and the effects of changes of the microscopic differential length can both be treated perturbatively. The index $j$ denotes any higher order mode transverse perturbations make excited.

\section{G. LIGO detector SB imbalance noise}

Unidimensional treatments preclude any side band imbalance when the system is locked and the dark port condition is satisfied. This same symmetry provides a cancellation of terms that the error signals of the length control system are constructed to fully exploit.

In fact, their nominal zero ideally coincides with the carrier operating point and since this is how the system locks, the side band symmetry and the working point are considered identifiable. This overall assumption is the historical reason for simulation results being symmetrized, with numerical programs being run for one side band only and the other believed identical. The first investigations of side band imbalance are due to William Kells; he made extensive studies of the power they build up and showed the side band resonant curves are distinct. They can be made overlap by tuning $\nu^{\text {mod }}$ and changing $l_{-}$from the microscopic carrier dark fringe value. In addition to this being not desirable, the overlap of the total power curves does not suffice to make the fields identical, which is clear, for example, when differential misalignment affects the arm input mirrors. We solve this problem for illustrative purposes but the mechanism is general and a similar analysis can be done for other perturbations. Since we want to show the consequences of the system extra variability with respect to the unidimensional description, a twodimensional model suffices. If energy is conserved

$$
\mathcal{R}_{\mathrm{arm}}^{\alpha}\left(\nu^{0}\right)=\exp \left[-i \bar{\alpha} \sigma_{2}\right] \sigma_{3} \quad \bar{\alpha}=\frac{\alpha}{\tan \eta}
$$

describes the carrier response to the arm input mirror misalignment. As before $\alpha=k w \theta$. For the recycling cavity $\mathbf{p}=\mathbb{1}$ and we assume $\mathcal{R}_{\mathrm{arm}}^{\alpha}\left(\nu^{0} \pm \nu^{\mathrm{mod}}\right)=$ $\exp \left[-i \alpha \sigma_{1}\right]$. The operator (13) is Hermitian. As a result $\mathcal{B P}\left(\delta^{0}\right)$ and its eigenvectors define a positive scalar product. Here $\delta^{0}=0$ satisfies our prescription for the carrier dark fringe as described in Sec. III. The following bright port operator

$$
\mathcal{B} \mathcal{P}\left(\delta^{0} \pm \delta^{\mathrm{mod}}\right)=\cos \delta^{\mathrm{mod}} \cos \alpha \mp \sigma_{1} \sin \delta^{\mathrm{mod}} \sin \alpha
$$

describes the persisting side band fields, expanded in the basis of eigenmodes of the carrier bright port operator. The selection determined by (14) is made nearly entirely recirculated by the recycling mirror. Its reflectivity is denoted by $r^{2}<1$. In LIGO $r \simeq \cos \delta^{\text {mod }}$ so that the dark port side band power is the total available for signal detection. The carrier light is also recirculated. Therefore

$$
\begin{gathered}
\psi_{\mathrm{DP}}^{\nu^{0}}=\frac{t \mathcal{D P}\left(\delta^{0}\right)}{1-r \mathcal{B} \mathcal{P}\left(\delta^{0}\right)} \psi_{\mathrm{IN}}=-i \sin \bar{\alpha} \sigma_{2} \frac{r \cos \bar{\alpha}+\sigma_{3}}{1-r^{2} \cos ^{2} \bar{\alpha}} \psi_{\mathrm{IN}} \\
\psi_{\mathrm{DP}}^{\nu^{0} \pm \nu^{\mathrm{mod}}}=\frac{t \mathcal{D} \mathcal{P}\left(\delta^{0} \pm \delta^{\mathrm{mod}}\right)}{1-r \mathcal{B} \mathcal{P}\left(\delta^{0} \pm \delta^{\mathrm{mod}}\right)} \psi_{\mathrm{IN}}= \pm i t \frac{\sin \delta^{\mathrm{mod}}\left[r \cos \delta^{\mathrm{mod}}-\cos \alpha\right] \pm \sigma_{1} \sin \alpha\left[r \cos \alpha-\cos \delta^{\mathrm{mod}}\right]}{\left(\cos \alpha-r \cos \delta^{\mathrm{mod}}\right)^{2}+\left(1-r^{2}\right) \sin ^{2} \alpha} \psi_{\mathrm{IN}} \\
k w=20.44 \times 10^{4} \quad \delta^{\mathrm{mod}}=15.07 \times 10^{-2} \quad t^{2}=28.05 \times 10^{-3}
\end{gathered}
$$




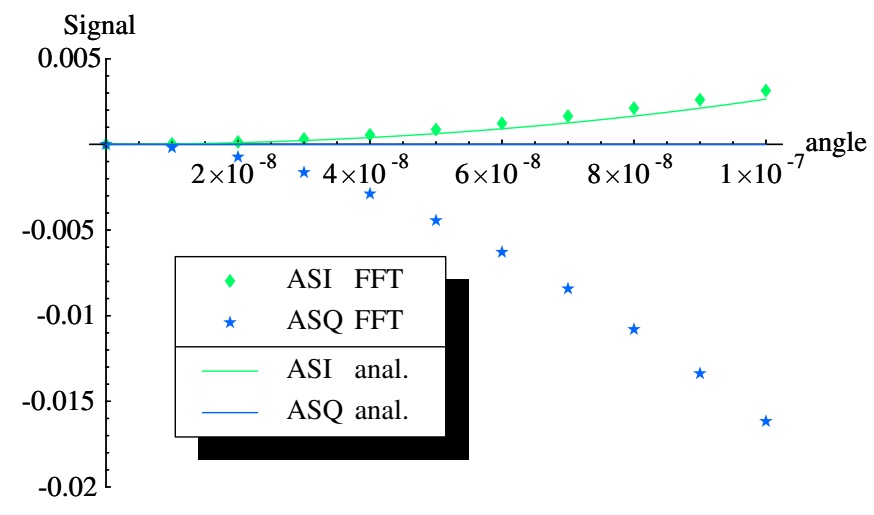

FIG. 20 (color online). Both simulations and analytical calculations assume two identical arm resonators and a perfectly matched recycling cavity; each run corresponds to a full LIGO interferometer lock.

where $t^{2}=1-r^{2}$ implies a lossless recycling mirror. The oscillating portion

$$
\left\langle\psi_{\mathrm{DP}}^{\nu^{0}-\nu^{\mathrm{mod}}} \mid \psi_{\mathrm{DP}}^{\nu^{0}}\right\rangle-\left\langle\psi_{\mathrm{DP}}^{\nu^{0}} \mid \psi_{\mathrm{DP}}^{\nu^{0}+\nu^{\mathrm{mod}}}\right\rangle
$$

of the output power is demodulated and two distinct signals are obtained, that constitute its two Fourier components. These are considered to be in phase and in quadrature with the input modulation signal (more generally with a phase of reference) and their form nominally corresponds to the imaginary and real part of (16). The \pm sign in (15) provides a cancellation that is ideal to extract the difference between the phases of the fields reflected form the arms. We evaluate (16) post-processing the numerical results of the FFT code.

The in-phase values of the demodulated antisymmetric port power are predicted by our analytical model

$$
\frac{2 i \sin \alpha \sin \bar{\alpha}}{1-r \cos \bar{\alpha}} \frac{t^{2}\left(\cos \delta^{\bmod }-r \cos \alpha\right)}{\left(\cos \alpha-r \cos \delta^{\bmod }\right)^{2}+\left(1-r^{2}\right) \sin ^{2} \alpha}
$$

which well describes the antisymmetric port in-phase (ASI) data points of Fig. 20. The antisymmetric port quadrature (ASQ) ones are the component in quadrature. For construction, ASQ is linearly sensitive to differential arm length changes and is in fact monitored by the LIGO control system, which actively keeps this signal at its null. On the contrary, there is no feedback between the ASI signal and any degree of freedom of the interferometer. Because it is predicted to be rigorously null by the unidimensional treatment, this signal is commonly ascribed to the finite accuracy of the locking system, through a power imbalance mechanism. Our analysis demonstrates the unavoidable character of this signal, which originates from the multidimensional nature of the intracavity optical fields.

\section{SUMMARY AND CONCLUSIONS}

In LIGO detectors, only one longitudinal degree of freedom can distinguish one side band from the other (as their frequency spacing is an integer multiple of the free spectral range of each cavity LIGO comprises) that is the macroscopic Schnupp asymmetry $l_{-} \ll \lambda^{\text {mod }}$. In ideal interferometers $l_{-}$is the only asymmetry of the system and its action is described by a scalar quantity; once this is made equivalent, the side bands are no longer distinguishable and all their components are the same. That is guaranteed by the carrier dark port condition and common perturbations do not change it. The separability of $l_{-}$is only precluded by differential perturbations that also make the definition of the dark port condition a tensorial problem. The relative matrix determines the modal content of the output field and mode selective solutions may or may not exist. In fact, while in the unidimensional case $l_{-}$can be independently adjusted, the optical modes of the system and the corresponding resonances are functions of $l_{-}$when the interferometer is not perfectly symmetric. This is therefore a nonlinear problem; ideally the fundamental mode and the dark port condition must be simultaneously determined. The governing operators must also be well-defined because free propagation is no longer a simple phase shift as in the unidimensional treatment of Sec. II. Hence in Sec. III we illustrate and justify our own prescription of the bright and dark port so that the corresponding operators can be consistently expanded in terms of the eigenmodes of the former.

Taking advantage of the form of the bright and dark port operators, a relation has been established, that simplifies the investigation of the complex problem of fundamental mode dark port condition. From it we derive in Sec. III the first analytical demonstration of a full correspondence relating the best approximate side band symmetry and the best approximate separability of $l_{-}$. We also demonstrate the level of these approximations being of third order in the strength of the differential perturbation, as long as perturbative methods are applicable. Examples are given in Sec. III that illustrate general and nongeneral features of optical differential perturbations and the limits of our perturbative approach.

The next level of complexity is the frequency dependence of the perturbations affecting the carrier and side bands, because this implies a different optimal $l_{+}$. This is explained in the first part of Sec. IV where we review the general concepts. The common configuration corresponds to Fig. 2 where the septum is mode selective. Other than in the case of Hermite-Gaussian or Laguerre-Gaussian cavity modes, any inserted optics are not phase matched to the entire family of standing wave solutions. These in fact must only have the same phase front for the resonator boundary, that is also the reason for the dark port to be identified at the same optical length from the input mirror as the bright port, which in turn coincides with the input 
mirror and defines the cavity modes. Note that the generalized Gouy phase can only be unambiguously defined for a set of modes with identical phase fronts. Our prescription relating the dark and bright port by symmetry is also ideal for recycling fundamental mode antisymmetric port signals, with the dark port field made to circulate back and forth between the system and the dark port.

In Sec. IV we logically build on our definitions of Sec. III. We find there is no unique operating point, satisfying the dark port condition and the side band equivalence. The former still corresponds to the solution of Sec. III identifying one fundamental mode with maximum power build up. However, the frequency dependence impact of the perturbations now violates the correspondence between carrier dark port condition and side band symmetry. The problem of differential tilt is studied again in the frequency dependent case. We compare the predictions of our model with the results of a simulation code and find an unavoidable nonzero ASI, defined as the in-phase component of the demodulated antisymmetric port power.

The gravitational wave signal is in quadrature to that and is denoted ASQ. The length control system of LIGO keeps this signal null, in order to maintain the working point of the interferometer and take advantage of its linear response to differential length changes. No feedback involves ASI and this contributes an unrestraint radio-frequency output signal which is collected by a sensitive photodetector and generates a current $\propto J_{0}(\Gamma) J_{1}(\Gamma) P_{\mathrm{IN}}$. The matched LIGO configuration nominally corresponds to $P_{\mathrm{IN}}=6 \mathrm{~W}$. Combining this with the modulation index $\Gamma \simeq 0.5$, we find $J_{0}(\Gamma) J_{1}(\Gamma) P_{\mathrm{IN}} \simeq 1 W$. This scaling factor multiplies the normalized data of Fig. 20 for ASI. The deposit of power is $\simeq 3.6 \mathrm{~mW}\left(\theta / 10^{-7} \mathrm{rad}\right)^{2}$ for purely differential tilt. The nature of this term is made manifest by our analytical form: it is contributed by higher order modes, for which the microscopic conditions the unidimensional treatment of Sec. II describes are not ideal. The operating point is a selective mode concept and ultimately applies to one single state system.
Eliminating parasitic modes implies engaging perfect optics, that must also be unaffected by any fundamental or instrumental noise source. The residual terms must then be actively suppressed, through either fundamental mode selection or neutralization of spurious signals.

For example, the introduction of an output mode cleaner would suppress the ASI optical term, whose practical relevance has led to the design and operation of an ASI servo. This system basically counteracts the photocurrent ASI generates and was first conceived and devised by Daniel Sigg when experimental evidence confirmed the predicted unavoidable nonzero ASI. The relative significance of ASI and ASQ is unfortunately enhanced by the high sensitivity of the detector, whose aim is measuring extremely small deviations of ASQ from its normal zero, which are recorded by very sensitive RF photodiodes, the optical power of ASI and ASQ is collectively released onto.

\section{ACKNOWLEDGMENTS}

This work was made possible by the support of Riccardo Desalvo, the assistance of Larry Wallace and his team and more generally the LIGO Laboratory, which is operating under the Cooperative Agreement No. PHY-0107417 of the National Science Foundation and the California Institute of Technology. One of us (E. D'A.) also thanks Daniel Sigg for his simple explanations of the complex response of LIGO.

\section{APPENDIX A: SUMMATION TECHNIQUES FOR ITERATED OPTICAL SCATTERING}

We assume the input beam coincides with the fundamental mode. Because of $l_{-}$, the side band higher order modes are not eliminated at the symmetric port and are made to recirculate by the input mirror. These parasitic modes therefore have the chance of being converted again into the fundamental mode as described by

$$
\begin{aligned}
\psi_{\infty}^{ \pm} \propto & \frac{1}{1-\cos \delta^{\bmod } \mathcal{B P}\left(\delta^{0}\right) \pm i \sin \delta^{\bmod } \mathcal{D} \mathcal{P}\left(\delta^{0}\right)} \psi_{\mathrm{IN}} \\
= & \frac{1}{1-\cos \delta^{\bmod } \mathcal{B} \mathcal{P}\left(\delta^{0}\right)} \psi_{\mathrm{IN}} \mp \frac{1}{1-\cos \delta^{\bmod } \mathcal{B} \mathcal{P}\left(\delta^{0}\right)} i \sin \delta^{\bmod } \mathcal{D} \mathcal{P}\left(\delta^{0}\right) \frac{1}{1-\cos \delta^{\bmod } \mathcal{B P}\left(\delta^{0}\right)} \psi_{\mathrm{IN}} \\
& -\frac{1}{1-\cos \delta^{\bmod } \mathcal{B P}\left(\delta^{0}\right)} \sin \delta^{\bmod } \mathcal{D} \mathcal{P}\left(\delta^{0}\right) \frac{1}{1-\cos \delta^{\bmod } \mathcal{B} \mathcal{P}\left(\delta^{0}\right)} \sin \delta^{\bmod } \mathcal{D} \mathcal{P}\left(\delta^{0}\right) \frac{1}{1-\cos \delta^{\bmod } \mathcal{B} \mathcal{P}\left(\delta^{0}\right)} \psi_{\mathrm{IN}}+\ldots
\end{aligned}
$$

where the interaction term

$$
\left[\mathbf{M}^{-1}\left(\delta^{0}\right) \sin \delta^{\bmod } \mathcal{D} \mathcal{P}\left(\delta^{0}\right) \mathbf{M}\left(\delta^{0}\right)\right]_{1 k} \times\left[\sum\right]_{k m}\left[\mathbf{M}^{-1}\left(\delta^{0}\right) \sin \delta^{\bmod } \mathcal{D} \mathcal{P}\left(\delta^{0}\right) \mathbf{M}\left(\delta^{0}\right)\right]_{m 1}
$$

depends on the series 


$$
\begin{aligned}
{\left[\sum\right]_{k m}=} & \frac{\delta_{k m}}{1-\cos \delta^{\bmod } \lambda_{k}\left(\delta^{0}\right)} \mp \frac{1}{1-\cos \delta^{\bmod } \lambda_{k}\left(\delta^{0}\right)}\left[\mathbf{M}^{-1}\left(\delta^{0}\right) i \sin \delta^{\bmod } \mathcal{D} \mathcal{P}\left(\delta^{0}\right) \mathbf{M}\right]\left(\delta^{0}\right)_{k m} \frac{1}{1-\cos \delta^{\bmod } \lambda_{m}\left(\delta^{0}\right)} \\
& -\frac{1}{1-\cos \delta^{\bmod } \lambda_{k}\left(\delta^{0}\right)}\left[\mathbf{M}^{-1}\left(\delta^{0}\right) \sin \delta^{\bmod } \mathcal{D} \mathcal{P}\left(\delta^{0}\right) \mathbf{M}\right]\left(\delta^{0}\right)_{k p} \frac{1}{1-\cos \delta^{\bmod } \lambda_{p}\left(\delta^{0}\right)}\left[\mathbf{M}^{-1}\left(\delta^{0}\right) \sin \delta^{\bmod } \mathcal{D} \mathcal{P}\left(\delta^{0}\right) \mathbf{M}\right] \\
& \times\left(\delta^{0}\right)_{p m} \frac{1}{1-\cos \delta^{\bmod } \lambda_{m}\left(\delta^{0}\right)}+\ldots
\end{aligned}
$$

where $k, m \neq 1$ and all summations are intended over indices $p, \ldots \neq 1$.

Note that the first order term is missing, as a consequence of the bright port condition and the relation with the dark port we have established in the text

$$
\begin{aligned}
\frac{\mathrm{d}}{\mathrm{d} \delta}\left[\mathbf{M}^{-1}(\delta) \mathcal{B P}(\delta) \mathbf{M}(\delta)\right]_{11}= & {\left[\frac{\mathrm{d} \mathbf{M}^{-1}(\delta)}{\mathrm{d} \delta} \mathcal{B P}(\delta) \mathbf{M}(\delta)+\mathbf{M}^{-1}(\delta) \frac{\mathrm{d} \mathcal{B} \mathcal{P}(\delta)}{\mathrm{d} \delta} \mathbf{M}(\delta)+\mathbf{M}^{-1}(\delta) \mathcal{B P}(\delta) \frac{\mathrm{d} \mathbf{M}(\delta)}{\mathrm{d} \delta}\right]_{11} } \\
= & {\left[\frac{\mathrm{d} \mathbf{M}^{-1}(\delta)}{\mathrm{d} \delta} \mathbf{M}(\delta)\right]_{1 m}\left[\mathbf{M}^{-1}(\delta) \mathcal{B P}(\delta) \mathbf{M}(\delta)\right]_{m 1}+\left[\mathbf{M}^{-1}(\delta) \frac{\mathrm{d} \mathcal{B} \mathcal{P}(\delta)}{\mathrm{d} \delta} \mathbf{M}(\delta)\right]_{11} } \\
& +\left[\mathbf{M}^{-1}(\delta) \mathcal{B P}(\delta) \mathbf{M}(\delta)\right]_{1 n}\left[\mathbf{M}^{-1}(\delta) \frac{\mathrm{d} \mathbf{M}(\delta)}{\mathrm{d} \delta}\right]_{n 1} \\
= & {\left[\frac{\mathrm{d} \mathbf{M}^{-1}(\delta)}{\mathrm{d} \delta} \mathbf{M}(\delta)\right]_{1 m} \delta_{m 1} \lambda_{1}(\delta)+\left[\mathbf{M}^{-1}(\delta) \frac{\mathrm{d} \mathcal{B} \mathcal{P}(\delta)}{\mathrm{d} \delta} \mathbf{M}(\delta)\right]_{11} } \\
& +\delta_{1 n} \lambda_{1}(\delta)\left[\mathbf{M}^{-1}(\delta) \frac{\mathrm{d} \mathbf{M}(\delta)}{\mathrm{d} \delta}\right]_{n 1} \\
= & {\left[\mathbf{M}^{-1}(\delta) \frac{\mathrm{d} \mathcal{B} \mathcal{P}(\delta)}{\mathrm{d} \delta} \mathbf{M}(\delta)\right]_{11}=-i\left[\mathbf{M}^{-1}(\delta) \mathcal{D} \mathcal{P}(\delta) \mathbf{M}(\delta)\right]_{11}=0 }
\end{aligned}
$$

and since the second order one is even, the violation of the side band balance is, at most, cubic. Quantitative assessments depend of course on the perturbation and the type of system the perturbation affects. Nonetheless, $\mathcal{S}^{ \pm}$is a relevant analytical quantity. It enables the estimation of the violation of the correspondence between dark port condition and side band symmetry and thus of the spurious terms shifting the error null.

\section{APPENDIX B: DIFFERENTIAL ABERRATION IN MICHELSON OPTICS}

Throughout the paper, we use oversimplified models to illustrate and interpret the consequences of the inseparability of $l_{-}$as demonstrated in the text. Here we want to show that the eigensolutions of (4) depend on $l_{-}$. For our discussion, a two-dimensional model suffices to reveal the fundamental differences between the unidimensional treatment and a state vector formalism. When more than the fundamental mode and its eigenvalue must be computed, a few transverse degrees of freedom ought be added to describe cross coupling among higher order modes. For illustrative purposes Fig. 21 and the related model are adequate enough. Unitarity is made manifest by the use of Pauli matrices. We address an example that we consider representative of most general situations, that is spheroidal deformations. The matrix $\exp \left[-i \theta^{G} \sigma_{3}\right]$ is the propagator and $\mathbf{m}(\alpha)=\exp \left[-i \alpha \sigma^{\prime}\right]$ represents the scattering action.
Here $\sigma^{\prime}=\sqrt{1 / 3} \sigma_{1}-\sqrt{2 / 3} \sigma_{3}$. Note that this type of perturbation has terms $\sim \alpha$ on the diagonal. The equation to be solved is

$$
\lambda_{1,2}(\delta) \psi_{i}(\delta)=\frac{\mathbf{p} e^{-i \delta} \mathbf{m}(\alpha) \mathbf{p}+\mathbf{p} e^{i \delta} \mathbf{m}(-\alpha) \mathbf{p}}{2} \psi_{i}(\delta)
$$

whose eigenvectors and eigenvalues

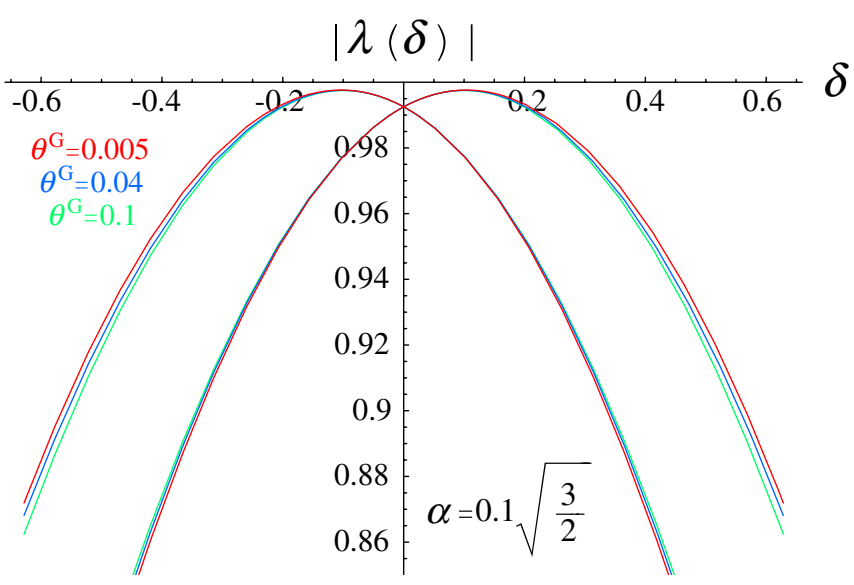

FIG. 21 (color online). The maxima of the absolute value of the two eigenvalues are different and each of them corresponds to a certain dark port condition. This feature is maintained in higher dimensions. 


$$
\begin{aligned}
\lambda_{i}= & \cos 2 \theta^{G} \cos \delta \cos \alpha-i \sin 2 \theta^{G} \sqrt{\frac{2}{3}} \sin \delta \sin \alpha \\
& \pm \sqrt{\Delta^{2}+\frac{1}{3} \sin ^{2} \delta \sin ^{2} \alpha} \\
\Delta= & \sqrt{\frac{2}{3} \sin \delta \sin \alpha \cos 2 \theta^{G}-i \cos \delta \cos \alpha \sin 2 \theta^{G}} \\
\psi_{1}(\delta)= & \left(\frac{\sqrt{3} \Delta}{\sin \delta \sin \alpha}-\sqrt{\left(\frac{\sqrt{3} \Delta}{\sin \delta \sin \alpha}\right)^{2}+1}\right) \\
\psi_{2}(\delta)= & \left(\frac{-\sqrt{3} \Delta}{\sin \delta \sin \alpha}+\sqrt{\left(\frac{\sqrt{3} \Delta}{\sin \delta \sin \alpha}\right)^{2}+1}\right.
\end{aligned}
$$

can be approximated as

$$
\begin{aligned}
& \lambda_{1,2} \simeq(\cos \delta \cos \alpha \pm \sqrt{2 / 3} \sin \delta \sin \alpha) \\
& \times \exp \left[\mp i \arctan \sqrt{\tan ^{2} 2 \theta^{G}-\frac{\tan ^{2} \delta \tan ^{2} \alpha}{3 \cos ^{2} 2 \theta^{G}}}\right] \\
& \psi_{1}(\delta) \simeq\left(\begin{array}{c}
1 \\
-i \sin \delta \sin \alpha /\left(2 \sqrt{3} \sin 2 \theta^{G}\right)
\end{array}\right) \\
& \psi_{2}(\delta) \simeq\left(\begin{array}{c}
i \sin \delta \sin \alpha /\left(2 \sqrt{3} \sin 2 \theta^{G}\right) \\
1
\end{array}\right)
\end{aligned}
$$

within the perturbative regime.

Set aside that more accurate predictions require more sophisticated models, it is clear that no differential length simultaneously satisfies the dark fringe condition for all eigensolutions.

\section{APPENDIX C: SENSITIVITY TO DIFFERENTIAL LENGTHS}

The nominally orthogonal ASI and ASQ channels have different responses to any change of the differential length. This is shown in Fig. 22 where the two sets of data correspond to the two output signals when additional changes $\delta l_{-}$are introduced. These overwrite the operating point the numerical simulations converge to and are added out of the locking routine (that is they are not further modified).

Note that a fraction of a nanometer would suffice to cancel ASQ. If redone for $L_{-}$a similar result would show

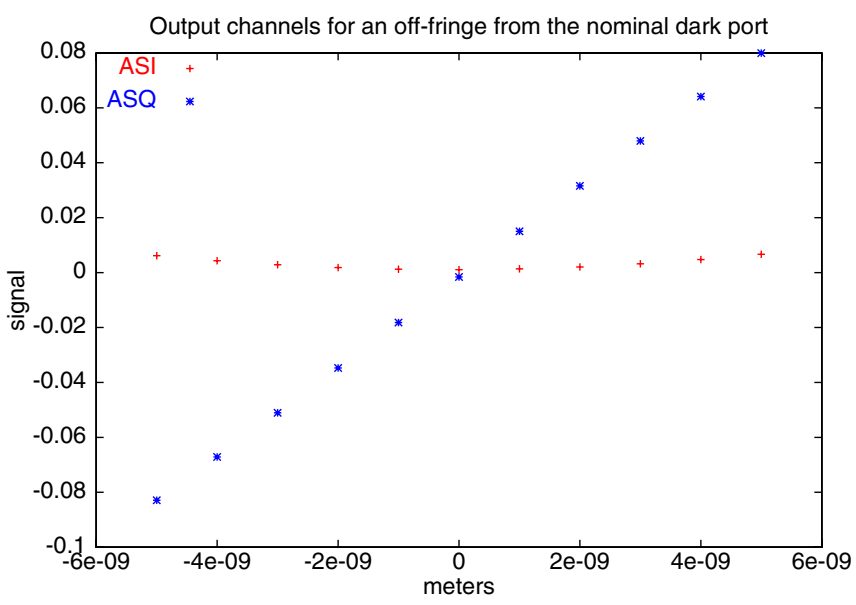

FIG. 22 (color online). The impact of small changes of the differential length is shown and a completely different response of ASI and ASQ is found. This is exactly what we expect and confirms that an accurate length control system would maintain ASQ at its nominal zero.

for changes of the order of picometers. When $l_{-}$changes Fig. 22 shows the quadratic response of ASI. These data illustrate the orthogonality of the two channels, confirm the unavoidable term the differential transverse perturbations contribute, and suggest an empirical method to distinguish ASI. The test done for Fig. 22 is the result of grid manipulation. The ASI and ASQ signals are obtained for $\theta=$ $10^{-7}$ rad differential tilt. Fields are $128 \times 128$ complex matrices which can be used to construct any signal.

Were the two actual channels as orthogonal as the two Fourier components they correspond to, the insensitive oscillating quantity could be observed for veto purposes, as it should not linearly respond to differential length changes and therefore to gravitational waves. If its amplitude and frequency were within the capacity of the control system, a differential excitation of the alignment of the arm input mirrors should be relatively more significant in the uncontrolled channel ASI and ideally reach a maximum at orthogonality. This would not solve the problem of the radio-frequency photodiode saturation. The transverse nature of the in-phase output signal makes ASI unavoidable, its contribution given by unsuppressed higher order modes. These can be relatively reduced by an output mode cleaner, as other forms of counteraction are limited by the input power.
[1] Ron Drever et al., Appl. Phys. B 31, 97 (1983).

[2] C. J. Walsh et al., Appl. Opt. 31, 2870 (1999).

[3] A.E. Siegman, Lasers (University Science Books, Sausalito, CA, 1986).

[4] D. Sigg et al., Appl. Opt. 40, 4988 (2001).
[5] Y. Hefetz, N. Mavalvala, and D. Sigg, J. Opt. Soc. Am. B 14, 1597 (1997); See also P960024 available from the LIGO Document Search Tool on http://www.ligo.org.

[6] R. Beausoleil and D. Sigg, J. Opt. Soc. Am. A 16, 2990 (1999). 
[7] R. Beausoleil et al., J. Opt. Soc. Am. B 20, 1247 (2003).

[8] M. Evans et al., Opt. Lett. 27, 598 (2002).

[9] A. Kostenbauder et al., J. Opt. Soc. Am. A 14, 1780 (1997).

[10] B. Bochner and Y. Hefetz, Phys. Rev. D 68, 082001 (2003).

[11] P. Fritschel et al., Appl. Opt. 37, 6734 (1998).

[12] D. E. McClelland et al., Opt. Lett. 24, 1014 (1999).

[13] A. Giazotto, Phys. Rep. 182, 365 (1989).

[14] C. W. Misner, J. A. Wheeler, and K. S. Thorne, Gravitation (Freeman, San Francisco, 1973).

[15] P. R. Saulson, Fundamentals of Interferometric Gravitational Wave Detectors (World Scientific, Singapore, 1994).

[16] J. B. Camp et al., J. Opt. Soc. Am. A 17, 1014 (2000).

[17] J. A. Sidles and D. Sigg, Phys. Lett. A 354, 167 (2006) and also as P030055 and T030120 from the LIGO Document Search Tool on www.ligo.org for an exhaustive treatment and interpretation.
[18] A. Gretarsson et al. (unpublished).

[19] From 03/25/2004 to 04/14/2004 available on Livingston Electronic Logs http://ilog.ligo-la.caltech.edu from the Detector web page.

[20] A.Gretarsson et al., L1 PRC studies 04/14/2004 informal talk for the initial LIGO commissioning meeting http:// ligo.mit.edu/commissioning/; and also T060104 from the LIGO Document Search Tool.

[21] E. D'Ambrosio 02/06/2004 and 02/20/2004 view graphs available from the TCS meeting group agenda at http:// www.ligo-wa.caltech.edu/ sigg/TCS/; and also T060103 and T060102 from the LIGO Document Search Tool.

[22] G. Billingsley for the as built parameters of all LIGO optics, from http://www.ligo.caltech.edu/ gari under link LIGO I Core Optics Components.

[23] D.Sigg (private communication); Hanford Electronic Logs read-only accessible at http://apex.ligo-wa.caltech.edu/ ilog/. 